\title{
Determining elastic anisotropy of textured polycrystals using resonant ultrasound spectroscopy
}

\author{
Jordan A. Evans ${ }^{1}$, Blake T. Sturtevant ${ }^{1}$ (1), Bjørn Clausen ${ }^{1}$ (D), Sven C. Vogel ${ }^{1}$ (i), \\ Fedor F. Balakirev ${ }^{1}$ (D), Jonathan B. Betts ${ }^{1}$, Laurent Capolungo ${ }^{1}$ (D), Ricardo A. Lebensohn ${ }^{1}$ (D), and \\ Boris Maiorov ${ }^{1, *}$ (1) \\ ${ }^{1}$ Los Alamos National Laboratory, Los Alamos, NM 87545, USA
}

Received: 27 August 2020

Accepted: 20 January 2021

Published online:

24 February 2021

(C) This is a U.S. government work and not under copyright protection in the U.S.; foreign copyright protection may apply 2021

\begin{abstract}
Polycrystalline materials can have complex anisotropic properties depending on their crystallographic texture and crystal structure. In this study, we use resonant ultrasound spectroscopy (RUS) to nondestructively quantify the elastic anisotropy in extruded aluminum alloy $1100-\mathrm{O}$, an inherently low-anisotropy material. Further, we show that RUS can be used to indirectly provide a description of the material's texture, which in the present case is found to be transversely isotropic. By determining the entire elastic tensor, we can identify the level and orientation of the anisotropy originated during extrusion. The relative anisotropy of the compressive $\left(c_{11} / c_{33}\right)$ and shear $\left(c_{44} / c_{66}\right)$ elastic constants is $1.5 \% \pm 0.5 \%$ and $5.7 \% \pm 0.5 \%$, respectively, where the elastic constants (five independent elastic constants for transversely isotropic) are those associated with the extrusion axis that defines the symmetry of the texture. These results indicate that the texture is expected to have transversely isotropic symmetry. This finding is confirmed by two additional approaches. First, we confirm elastic constants and the degree of elastic anisotropy by direct sound velocity measurements using ultrasonic pulse echo. Second, neutron diffraction (ND) data confirm the symmetry of the bulk texture consistent with extrusioninduced anisotropy, and polycrystal elasticity simulations using the elastic selfconsistent model with input from ND textures and aluminum single-crystal elastic constants render similar levels of polycrystal elastic anisotropy to those measured by RUS. We demonstrate the ability of RUS to detect texture-induced anisotropy in inherently low-anisotropy materials. Therefore, as many other common materials have intrinsically higher elastic anisotropy, this technique should be applicable for similar levels of texture, providing an efficient general diagnostic and characterization tool.
\end{abstract}

Handling Editor: P. Nash.

Address correspondence to E-mail: maiorov@lanl.gov 


\section{Introduction}

Most polycrystalline materials show some degree of anisotropy. In general, anisotropy may arise in these materials from the crystal symmetry of the material compounded with a distribution of crystallographic orientations of the single-crystal grains in the aggregate (i.e., texture), and/or by an inhomogeneous distribution of secondary phases [1]. Defects generated by thermomechanical processes can also produce elastic anisotropy if they exhibit a preferential alignment or orientation [2]. Anisotropic properties have profound influence on many properties, such as a material's response to mechanical or magnetic stimuli, and can be exploited to design materials with directionally preferential properties [3-5].

Crystallographic texture can result from a variety of common manufacturing and forming processes, such as extruding, swaging, rolling and cold working [6-12]. Texture can be characterized by several distinct methods, including X-ray diffraction (XRD), electron backscatter diffraction (EBSD) and neutron scattering [4, 13-18]. However, these methods are either limited to characterizing texture on the surface of the material, or require large user facilities with competitive access.

Alternatively, acoustic characterization techniques offer the possibility of probing the bulk of a material as mechanical waves travel through it, as is the case for the pulse-echo ultrasonic technique [19]. In particular, resonant ultrasound spectroscopy (RUS) is a fast, high-resolution, nondestructive characterization technique whereby the mechanical resonance frequencies of a specimen can be measured and used to determine the elastic properties of a material. RUS, nonlinear RUS and pulse-echo characterization constitute a powerful arsenal of nondestructive characterization techniques [20, 21]. The mechanical resonance frequencies of a material are set by the sample's geometry, mass, internal symmetry, and elastic constants. Using these parameters, one can obtain the complete elastic tensor by solving the inverse problem [22]. This can be accomplished by using a nonlinear iterative Levenberg-Marquardt algorithm, which minimizes the deviation between the measured and predicted values of resonance frequencies based on the material's elastic constants [23-27]. Historically, there have been attempts to use RUS to quantify the elastic anisotropy induced by texture. However, the symmetry selection process and the results obtained were not fully validated against texture measurements from other methods $[28,29]$. Foster et al. [30] used RUS to determine the elastic anisotropy of rolled $\mathrm{Cu}-\mathrm{Zn}$ (brass) alloys in combination with analytical expressions derived from polycrystal models based on simple averaging procedures to obtain a relatively compact representation of the sample's texture in terms of principal orientation distribution coefficients for comparison with texture measurements by neutron diffraction (ND). A related approach, but using new RUS and ND measurements on $\mathrm{Al}$ polycrystalline samples and more sophisticated modeling tools, is used in this work. More recently, Lan et al. [28, 29] proposed a new technique to directly measure the texture of a material using acoustic waves and contrasted this technique against resonant ultrasound spectroscopy (RUS) and neutron diffraction. Similarly, the effect of density of defects has been studied by multiple ultrasonic and microstructural characterization methods in aluminum and other material [20, 21].

The objective of the present work is to assess the potential for RUS to identify the symmetry and orientation of the anisotropy of a polycrystalline aluminum sample, as well as to quantify the degree of anisotropy. The focus of the present work is placed on indirectly quantifying the symmetry of the crystal orientation distribution rather than providing a full texture representation. Aluminum is an inherently low-anisotropy material and was selected with the intention of testing the lower limits of detection possible using RUS. Application of the RUS method on extruded aluminum samples clearly suggests that the material texture is transversely isotropic.

Further, we validate RUS results with direct measurements using ultrasonic pulse-echo technique, as well as crystallographic texture measurements by neutron diffraction combined with elastic self-consistent (ELSC) micromechanical simulations that give the polycrystal elastic constants as a function of the known single-crystal elastic constants and the texture of the sample. The ultrasonic pulse-echo technique (PE) is another method that can be used to determine the elastic properties of a material. In PE, the sound velocity of the material is measured along different orientations [31-33]. Because the sound velocity is extracted directly from the time-of-flight, PE results are not subject to fitting or convergence. For lowersymmetry materials, however, PE cannot determine 
all of the elastic constants in a single measurement. Independent measurements in different orientations with respect to the anisotropy of the sample are required [34, 35]. The consequent sample manipulation may introduce variations in delicate samples, or result in complications when characterizing hazardous samples. These ultrasonic experiments are performed in the anelastic limit, i.e., the response is elastic (linear) and non-hysteretic. The level of losses is minimal but measurable, allowing RUS and PE the capability to extract changes in sound attenuation (internal friction). As a result, the measurements are nondestructive and samples are unchanged. PE measurements are found to be in very good agreement with RUS characterization. Finally, the ELSC predictions of the material's effective stiffness tensor, which utilizes the materials' bulk texture as measured by neutron diffraction, shows very good, but not perfect, agreement with those measured by RUS, thereby demonstrating that the RUS can be used to extract key features in the elastic properties of strongly textured materials.

\section{Elastic response of a solid, anisotropy and relationship to wave velocities}

The effective elastic response of a solid, as described by Hooke's law in Eq. 1, linearly relates the Cauchy stress, $\sigma_{i j}$, to the elastic strain, $\varepsilon_{k l}$, by the elastic stiffness tensor $c_{i j k l}$. In the most general anisotropic case, the stiffness tensor has 21 independent constants due to the symmetries associated with stresses and strains and the uniqueness of strain potential energy. Alternatively, Hooke's law can be written in matrix form using Voigt notation in Eq. 2:

$$
\begin{aligned}
& \sigma_{i j}=c_{i j k l} \varepsilon_{k l} \\
& c_{i j k l}=c_{j i k l}=c_{i j l k}=c_{j i l k}=c_{k l i j} \\
& {\left[\begin{array}{l}
\sigma_{11} \\
\sigma_{22} \\
\sigma_{33} \\
\sigma_{23} \\
\sigma_{13} \\
\sigma_{12}
\end{array}\right]=\left[\begin{array}{llllll}
c_{11} & c_{12} & c_{13} & c_{14} & c_{15} & c_{16} \\
& c_{22} & c_{23} & c_{24} & c_{25} & c_{26} \\
& & c_{33} & c_{34} & c_{35} & c_{36} \\
& & & c_{44} & c_{45} & c_{46} \\
& \text { sym } & & & c_{55} & c_{56} \\
&
\end{array}\right]\left[\begin{array}{c}
\varepsilon_{11} \\
\varepsilon_{22} \\
\varepsilon_{33} \\
2 \varepsilon_{23} \\
2 \varepsilon_{13} \\
2 \varepsilon_{12}
\end{array}\right]}
\end{aligned}
$$

For polycrystalline materials with no texture, each grain is randomly oriented such that the bulk elastic properties are equal in all directions. In this isotropic case, the stiffness tensor only has two independent constants, i.e., two degrees of freedom (DoF), and reduces to Eq. 3 where $c_{12}=c_{11}-2 c_{44}$. For materials with cubic symmetry, the stiffness tensor has three independent elastic constants, whereby $c_{12}$ is independent of $c_{44}$ and $c_{11}$.

$$
C_{i j}=\left[\begin{array}{cccccc}
c_{11} & c_{12} & c_{12} & 0 & 0 & 0 \\
& c_{11} & c_{12} & 0 & 0 & 0 \\
& & c_{11} & 0 & 0 & 0 \\
& & & c_{44} & 0 & 0 \\
& \text { sym } & & & c_{44} & 0 \\
& & & & & c_{44}
\end{array}\right]
$$

The effective values of $c_{11}$ and $c_{44}$ for a polycrystalline material depend on the single-crystalline counterparts. There are different approximations to calculate this relationship, the most common of which are the Voigt [36, 37] and Reuss [36, 37] averages obtained by assuming homogenous strain or stress, respectively, which, respectively, provide the upper and lower bounds for the polycrystal's elastic constants estimates. Alternatively, the Hashin and Shtrikman method which is based on a variational approach can also provide bounds [38, 39]. Finally, the elastic self-consistent (ELSC) model, originally proposed by Hershey [40] for aforementioned case of polycrystalline aggregates of randomly oriented single crystals with cubic symmetry, can provide a unique estimate of the homogenized elastic response of the medium [40]. These models can be generalized to aggregates of arbitrary single-crystal symmetry and non-uniform texture, in which case the polycrystal elastic constants will depend of the singlecrystal anisotropy and the orientation distribution of the grains. The recent advances obtained in mathematical methods used to extract the crystallographic texture from ultrasonic response, even for hexagonal symmetries, make determining the elastic anisotropy even more relevant [41].

A measure of intrinsic anisotropy in cubic materials is given by Zener's elastic anisotropy factor, $A$, in Eq. 4 [42]. Almost all crystalline materials have $A \neq 1$; when those materials form polycrystalline aggregates, texture means that their physical properties are orientation-dependent. Aluminum has a cubic crystal structure with a relatively small Zener factor of $A=1.22$ [43]. Aluminum is notably less anisotropic than most other cubic metals, such as copper $(A=3.21)$ [44] and 304 stainless steel (SS) $(A=3.77)$ [45], for example. 


$$
A=\frac{2 c_{44}}{c_{11}-c_{12}}
$$

For isotropic materials $(A=1)$, the Young's modulus $E$ (stiffness under uniaxial tension), bulk modulus $B$ (resistance to volumetric changes), shear modulus $\mu$ (stiffness under shear deformation) and Poisson ratio $v$ (ratio of transversal strain to axial strain), also referred to as the engineering constants, are given by the relations in Eqs. 5, 6, 7, and 8.

$E=\frac{c_{11}^{2}+c_{11} c_{12}-2 c_{12}^{2}}{c_{11}+c_{12}}$

$B=\frac{c_{11}+2 c_{12}}{3}$

$\mu=c_{44}$

$v=\frac{c_{12}}{c_{11}+c_{12}}$

If a manufacturing or forming process produces texture with axial anisotropy, as is the case for extrusion, the material will have transversely isotropic symmetry [46-50]. In the transversely isotropic case, the stiffness tensor has five independent constants (i.e., the same number than in a hexagonal symmetry), as shown in Eq. 9 and the orientation has been chosen such that the " $\mathrm{z}$ " axis is parallel to the texture axis (parallel to the rod's extrusion direction). As a result of the transversely isotropic texture, the values of $E, B, \mu$ and $v$ depend on orientation. The engineering constants for a transversely isotropic solid parallel $(||)$ and perpendicular $(\perp)$ to the axis of symmetry are given in Eqs. 10, 11, 12 and 13 [51-53].

$c_{i j}=\left[\begin{array}{cccccc}c_{11} & c_{12} & c_{13} & 0 & 0 & 0 \\ & c_{11} & c_{13} & 0 & 0 & 0 \\ & & c_{33} & 0 & 0 & 0 \\ & & & c_{44} & 0 & 0 \\ & \text { sym } & & & c_{44} & 0 \\ & & & & & \frac{1}{2}\left(c_{11}-c_{12}\right)\end{array}\right]$

$E_{\perp}=\frac{c_{11}^{2} c_{33}+2 c_{13}^{2} c_{12}-2 c_{13}^{2} c_{11}-c_{12}^{2} c_{33}}{c_{11} c_{33}-c_{13}^{2}}$

$E_{\|}=\frac{c_{11} c_{33}+c_{12} c_{33}-2 c_{13}^{2}}{c_{11}+c_{12}}$

$B=\frac{2 c_{11}+c_{33}+2\left(2 c_{13}+c_{12}\right)}{9}$

$$
\begin{aligned}
& \mu_{23}=\mu_{13}=c_{44} \\
& \mu_{12}=c_{66}=\frac{1}{2}\left(c_{11}-c_{12}\right) \\
& v_{\perp}=\frac{c_{12} c_{33}-c_{13}^{2}}{c_{11} c_{33}-c_{13}^{2}} \\
& v_{\| \perp}=\frac{c_{11} c_{13}-c_{12} c_{13}}{c_{11}^{2}-c_{12}^{2}} \\
& v_{\perp \|}=\frac{c_{11} c_{13}-c_{12} c_{13}}{c_{11} c_{33}-c_{13}^{2}}
\end{aligned}
$$

The relationships between the phase velocities of ultrasonic plane waves and the elastic constants of a material can be determined as shown in detail in the Appendix. For isotropic materials (two independent elastic constants), $c_{44}=\rho v_{T}^{2}$ and $c_{11}=\rho v_{L}^{2}$, where $\rho$ is the density, $v_{T}$ is the transverse wave velocity, and $v_{L}$ is the longitudinal wave velocity. For materials with lower symmetries, such as the transversely isotropic case (five independent elastic constants), determining the relationships between sound velocities and elastic constants is far more complicated, as shown in the Appendix.

\section{Experimental and modeling methods}

\section{Sample preparation}

All of the textured samples were machined from the same extruded rod of the commercial aluminum alloy (AA) 1100. AA-1100, also known as commercially pure aluminum, is composed of $>99 \%$ aluminum with silicon and iron as its primary impurities. The AA-1100 rod was provided in the annealed condition with no cold working (type $\mathrm{O}$ temper) in accordance with ASTM B221-14 standards. The AA-1100-O rod is assumed to have transversely isotropic texture as a result of the extrusion process during manufacturing [6-8]. An illustration of the transversely isotropic AA-1100-O rod is shown in Fig. $1 \mathrm{~b}$ from whence rectangular parallelepiped resonators (RPRs) were machined with sides $a, b$, or $c$ parallel to the rod axis. RPRs are advantageous for RUS experiments in comparison with other geometries (cylinders, cubes or spheres) because they have no degenerate resonance modes when the dimensions are correctly chosen [22, 27]. The flat parallel faces in principal directions also make RPRs suitable for pulse-echo experiments. Several RPRs were 
Figure 1 a, c RUS

experimental setup with

sample. $\mathbf{b}$ Illustration of 1100

rod with $\widehat{z}$-axis chosen parallel to the extrusion-induced axis of texture and orientations of RPR samples machined from the rod relative to the texture axis.
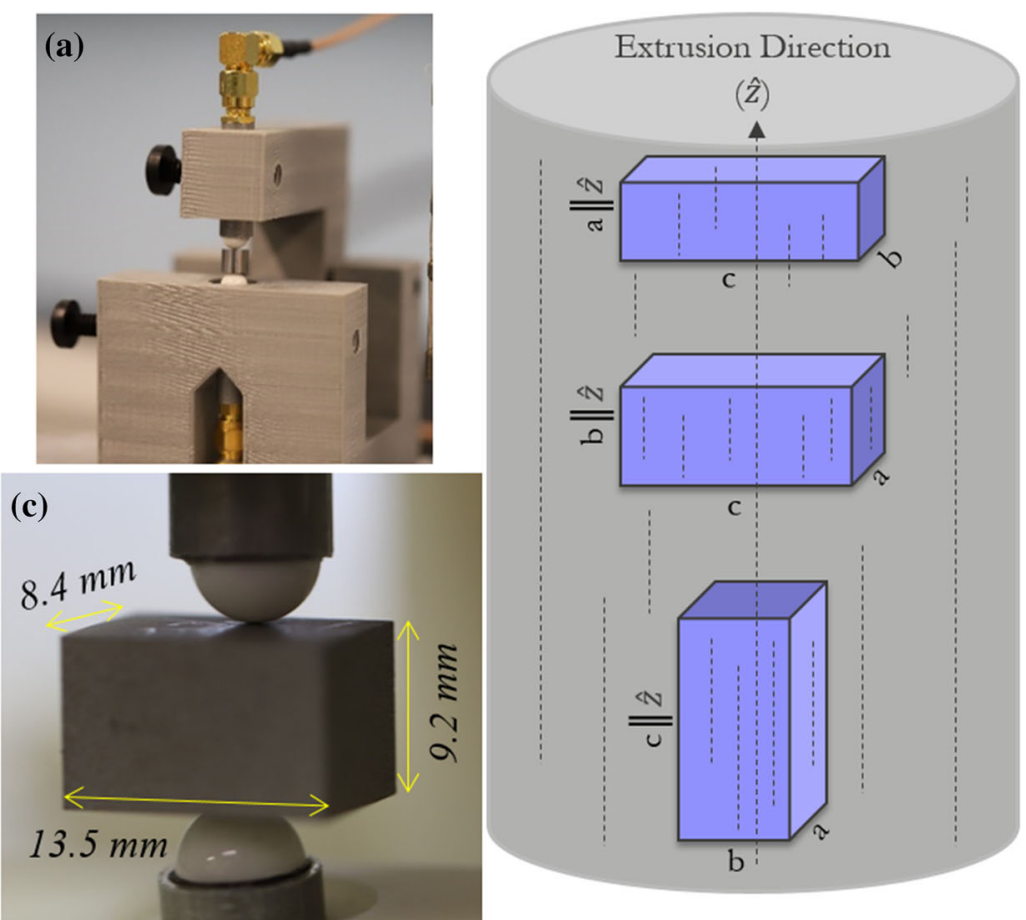

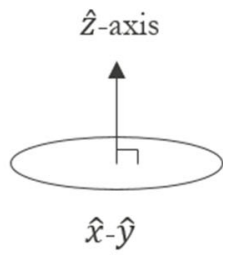

plane of

symmetry machined from this rod with dimensions shown in Fig. 1b, where $a=8.4 \mathrm{~mm}, \quad b=9.2 \mathrm{~mm}$ and $c=13.5 \mathrm{~mm}$ with a precision of $0.0006 \mathrm{~mm}$. To simplify distinguishing the texture orientation of the samples machined, we define $\widehat{z}$ as the direction parallel to the axis of extrusion. All samples were machined by electrical discharge machining (EDM) using skim cut settings in order to minimize surface roughness and damage caused by machining [54-56]. The AA-1100-O RPRs were then heat-treated at $615 \mathrm{~K}$ for $1 \mathrm{~h}$ in accordance with ASM standards in order to anneal defects present in the sample [57]. The AA1100-O RPRs have a density of $2.6899 \pm 0.0034 \mathrm{~g} \mathrm{~cm}^{-3}$, calculated from measuring geometrical volume and mass using a high-precision micrometer and scale. Thus, these samples are $99.26 \%$ dense, assuming a theoretical density of $2.71 \mathrm{~g} \mathrm{~cm}^{-3}$.

\section{Resonant ultrasound spectroscopy}

The RUS system was constructed at Los Alamos National Laboratory, the general description of which is described elsewhere [24, 58]. In Fig. 1a, c, we can observe the experimental setup used. The RUS system interfaces with the computer via a field-programmable gate array-based Red Pitaya detector system, which is programmed to generate the excitation signal to one transducer as well as record the response signal from the other transducer. The RUS software was developed at Los Alamos National Laboratory and is freely available online [58].

The driving and detecting transducers are identical and are composed of an aluminum housing filled with epoxy, a coaxial SMA connector on the one end, and a 5-mm-diameter lead zirconate titanate (PZT) piezoelectric transducer glued to the flat portion of an alumina hemisphere as described elsewhere and shown in Fig. 1a, c [59-61]. The alumina hemispherical cap not only provides electrical insulation for the piezoelectrics when measuring metallic samples, but also protects the piezoelectrics from physical damage while simultaneously guaranteeing excellent point contact with the sample. This allows the sample to resonate freely without having to balance it precariously by its corners. It also allows to reproduce the position of the sample with respect to the transducers. The resonance frequencies of the specimens are independent of the positioning or orientation of the sample between the driving and detecting transducers; in practice, however, some resonances may have undetectably low amplitudes which depend on positioning (e.g., if the detecting transducer is in contact with the sample which coincides with the node of a particular resonance). We found that placing the sample vertically between the transducers (i.e., parallel to the $c$ dimension) allowed the 
detection of at least 30 of the first 35 resonances. This sample arrangement was used for all the samples in order to reduce the small variations associated with measuring resonances in different positions on the sample. This arrangement, combined with the optimized sample geometry, yields easily detectable/ distinguishable resonances with large contributions from shear, dilatational and compressive elastic constants. In turn, the extracted resonance frequencies result in an overdetermined problem for the extraction of all the elastic constants from the measured resonance frequencies. The quality factors $(Q-$ values, the frequency-to-bandwidth ratio, defined by the resonance frequency divided by its full-width at half-maximum) of the resonances vary, but most resonances were measured with $Q$-values exceeding 20,000 as shown in Fig. 2.

The RUS inverse problem fitting procedure was executed using 12-order polynomials for all fits, although no significant differences were obtained using 14-order polynomials (deviations smaller than $0.02 \%$ ). The results obtained in the RUS fits are insensitive to the initial input elastic constant values. We used more than 30 of the first 35 resonance frequencies in the fitting procedure for each sample [24]. The fits assume a particular symmetry, set by the degrees of freedom and orientation selected in the fit $[22,24]$. The ability to extract the elastic constants of a polycrystalline material requires matching the symmetry of the fit with that of the sample. Extruded aluminum has transversely isotropic texture, which

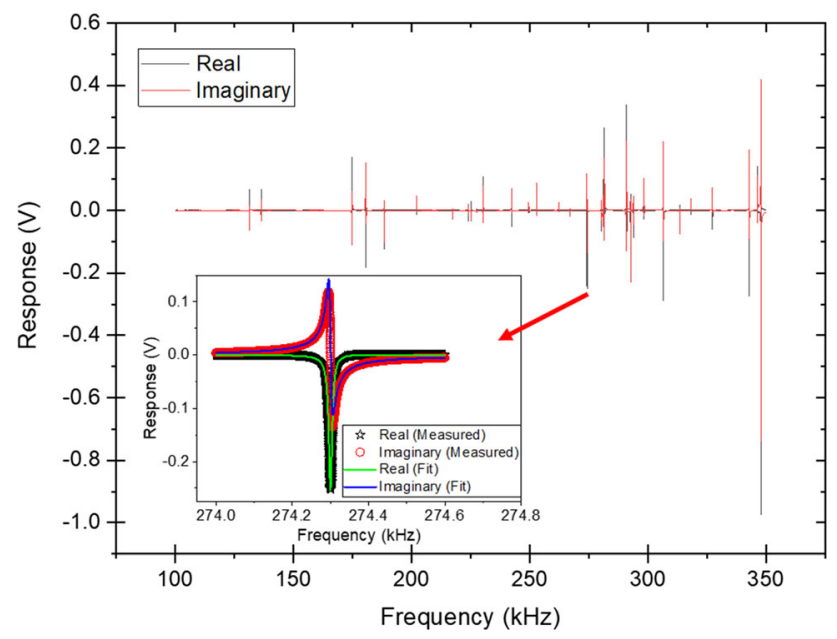

Figure 2 Measured RUS spectrum for an AA-1100-O RPR with a magnified view of the resonance at $274.3 \mathrm{kHz}$ and the Lorentzian fit overlaid. results in hexagonal symmetry (in the single-crystal sense). In order to distinguish physical orientations of the sample $\mathbf{a}, \mathbf{b}$ and $\mathbf{c}$ (as described in the previous section and illustrated in Fig. $1 \mathrm{~b}$ with the fit orientations involved in the RUS algorithm, we define the orientation of a hexagonal axis of symmetry in the fit to be $\boldsymbol{h}$. The fit should only be successful if its orientation matches the physical orientation of the sample's anisotropy (i.e., $\boldsymbol{h}|| \widehat{z}$ for transverse isotropy). Similarly, as shown later in the paper, the symmetry and orientation can be determined by solving the inverse problem with a lower symmetry.

\section{Ultrasonic pulse-echo}

The conventional pulse-echo time-of-flight technique was used to determine the speed of compressive and shear ultrasonic waves along all principal axes of the samples in order to directly obtain elastic constants for validation purposes [34, 35]. The experimental setup consisted of an arbitrary function generator and digitizing oscilloscope connected to an ultrasonic transducer, fused silica buffer rod and sample as described elsewhere [62, 63]. The electrical signal applied to the transducer was a tone burst with a Tukey envelope applied to mitigate spurious highfrequency effects [62, 64]. For improved accuracy in the determined time-of-flight, measurements were performed over a range of frequencies, typically $\sim 10 \mathrm{MHz}$ and $\sim 20 \mathrm{MHz}$ for shear and compressive modes, respectively. Measurement over a range of frequencies enables a frequency-dependent correction to be applied to account for the medium used to couple the buffer rod to the sample as previously described [62]. The accuracy of measured time-of-flight $(\tau)$ and physical dimensions $(d)$ and thus the sound speeds $(v=2 d / \tau)$ were of order $10^{-4}$ $10^{-3}$.

Because the same samples were used for the RUS and the PE experiments, and the transducer must be adhered to a flat surface in the PE experiments, PE experiments could only be performed for $\theta=0^{\circ}$ and $\theta=90^{\circ}$ relative to $\widehat{z}$ (see Appendix). With this orientation constraint and using Eq. 21, the PE experiments were used to determine $c_{11}, c_{33}, c_{44}$ and $c_{66}$, while $c_{13}$ could not be measured since its only contribution to the sound velocity comes from the $\sin ^{2} 2 \theta$ component of Eq. 20 (see the Appendix). In order to measure $c_{13}$ in rectangular parallelepiped samples machined from this textured rod, the faces of the 
sample need to be oriented, for example, at $45^{\circ}$ with respect to $\widehat{z}$.

\section{Neutron diffraction}

Aluminum is an inherently low-anisotropy material $(A=1.22)$. As such, the degree of texture required (measured in multiple of random density (MRD)) in order to result in measurable changes in its elastic properties is expected to be large. Bulk extrusionproduced texture was measured using time-of-flight neutron diffraction on the high-pressure preferred orientation time-of-flight diffractometer (HIPPO) $[65,66]$ at the pulsed spallation neutron source at the Los Alamos Neutron Science Center (LANSCE) of Los Alamos National Laboratory [67]. HIPPO utilizes $1200{ }^{3} \mathrm{He}$ detector tubes arranged on 45 detector panels on five rings with nominal diffraction angles of $40^{\circ}, 60^{\circ}, 90^{\circ}, 120^{\circ}$ and $140^{\circ}$, covering $22.4 \%$ of the $4 \pi$ steradians around the sample [68]. The RPRs were mounted on cadmium-wrapped sample holders as described elsewhere [65]. The mounted samples were loaded on the HIPPO sample changer robot [69], and neutron diffraction data were collected at rotation angles of $0^{\circ}, 67.5^{\circ}$ and $90^{\circ}$ for $10 \mathrm{~min}$ per rotation, covering $51.7 \%$ of the $4 \pi$ steradians [68]. The measured diffraction patterns were analyzed using the Materials Analysis Using Diffraction (MAUD) software [70] via simultaneous Rietveld refinement [71] of 132 neutron time-of-flight diffraction patterns following procedures described elsewhere [72]. An EWIMV representation of $7.5^{\circ}$ resolution was used to represent the orientation distribution function (ODF) in MAUD. No specific texture symmetry was assumed in the refinement. Lastly, in order to generate input for ELSC calculations of polycrystal elastic constants, the ODF was integrated in small, regularly spaced (every $10^{\circ}$ ) volumes of orientation space $\left(\varphi_{1}, \cos \Phi, \varphi_{2}\right)$, where $\left(\varphi_{1}, \Phi, \varphi_{2}\right)$ are the Euler angles (Bunge convention), resulting in a set of 23,328 discrete orientations centered inside each volume, determining a $72 \times 18 \times 18$ partition of $\varphi_{1}, \cos \Phi, \varphi_{2}$ in the intervals $[0,2 \pi],[0,1]$ and $[0, \pi / 2]$, respectively, with associated weights corresponding to the ODF integrals inside each volume.

\section{Elastic self-consistent model}

Self-consistent (SC) homogenization methods are used to estimate the mechanical response behavior of polycrystals, based on the knowledge of the properties, morphology and orientation distribution of the constituent single-crystal grains. The ELSC method was proposed independently by Hershey [37] and generalized by Hill [73]. Here, we use the most recent ELSC numerical implementation by Tomé and Lebensohn [74]. In the context of the SC theory, the polycrystal is represented by a set of weighted crystal orientations. The orientations represent grains, and the weights represent volume fractions. The set of orientations and weights are chosen to reproduce the crystallographic texture of the material. In this regard, the ELSC model utilizes the whole texture information contained in the full ODF measured by ND, instead of reduced representations, e.g., orientation distribution coefficients used by Foster et al. [30] for cubic materials, or texture coefficients for lower-symmetry crystals (e.g., [75] for hexagonal materials).

The ELSC model is a mean-field approximation based on 1-point statistics, in which each grain is treated as an ellipsoidal elastic inhomogeneity embedded in an effective homogenized elastic medium. The ellipsoidal shape represents the average morphology of the grains. The inhomogeneity character derives from the difference in elastic properties of each individual grain and the effective medium. The inhomogeneity and the medium will generally have fully anisotropic properties, deriving from the intrinsic single-crystal anisotropy, crystallographic texture and/or non-equiaxed morphology of the grains. The effective medium represents the average environment surrounding each grain. Using the equivalent Eshelby's inclusion method [76] to solve each heterogeneity problem, the ELSC estimate for the polycrystal's elastic stiffness (in tensorial notation) is given by the expression:

$c_{i j k l}=\left\langle c_{i j p q}^{g} A_{p q k l}^{g}\right\rangle$

where $c_{i j k l}$ and $c_{i j k l}^{g}$ are the elastic stiffness tensors of the polycrystal and each individual grain, the symbol $<$. $>$ indicates weighted average over the set of grains representing the aggregate, and $A_{i j k l}^{g}$ is the localization tensor associated with each grain $(g)$, which relates the strain applied to the polycrystal and the local strain in the grains, i.e., $\varepsilon_{i j}^{g}=A_{i j k l}^{g} \varepsilon_{k l}$ and is defined by: 
$A_{i j k l}^{g}=\left(c^{g}+\tilde{c}\right)_{i j p q}^{-1}(c+\tilde{c})_{p q k l}$

where $\tilde{c}_{i j k l}$ is the interaction tensor that gives the local deviations in stress in terms of the local deviations in stresses, i.e., $\sigma_{i j}^{g}-\sigma_{i j}=-\tilde{c}_{i j k l}\left(\varepsilon_{k l}^{g}-\varepsilon_{k l}\right)$, and is given by:

$\tilde{c}_{i j k l}=c_{i j m n}(I-S)_{m n p q} S_{p q k l}^{-1}$

where $S_{i j k l}$ is the Eshelby tensor [77], a function of $c_{i j k l}$ and the shape of the ellipsoid representing the morphology of the grains [76]. Given these dependencies, Eq. (14) is a fix-point equation that allows obtaining $c_{i j k l}$ iteratively.

Note that Eq. (14) involves two weighted averages of the elastic moduli of each grain to give the polycrystal's stiffness tensor. The first set of weights are the (tensorial) factors given by the localization tensors (Eq. 15), which depend on the relative stiffness and interaction between each grain and the effective medium representing the polycrystal. The second set of weights are the normalized values of the ODF integrals inside each of 23,328 volumes in orientation space, which provide the dependence with the polycrystal's texture.

\section{Results and discussion}

In order to determine the errors associated with an isotropic fit (2 DoF) of an RUS spectrum for an elastically isotropic sample, a $99.95 \%$ pure polycrystalline tungsten RPR was measured using RUS and the elastic constants were determined using both isotropic (2 DoF) and orthorhombic (9 DoF) fits.

Tungsten was chosen because its elastic properties are inherently isotropic ( $A=1.004$ for single-crystal tungsten) [78]. This guarantees isotropic elastic properties irrespective of the polycrystal's texture, because the elastic contributions of each grain are independently isotropic. RPRs of tungsten were machined using skim EDM with dimensions of $8.4 \pm 0.0012 \mathrm{~mm} \times 9.2 \pm 0.0015 \mathrm{~mm} \times 13.5 \pm 0.001$ $\mathrm{mm}$ resulting in a density of $19.135 \pm 0.006 \mathrm{~g} \mathrm{~cm}^{-3}$, $99.2 \%$ that of the $19.3 \mathrm{~g} \cdot \mathrm{cm}^{-3}$ nominal density [56].

The elastic constants determined by these two fitting methodologies, shown in Fig. 3, are identical (within error $\sim 0.05 \%$ ). These results show that a root-mean-square (RMS) error of $0.1 \%$ or lower can be achieved in RUS measurements if the sample geometry and microstructure are representative of the fit used, i.e., sharp $90^{\circ}$ corners and flat faces for

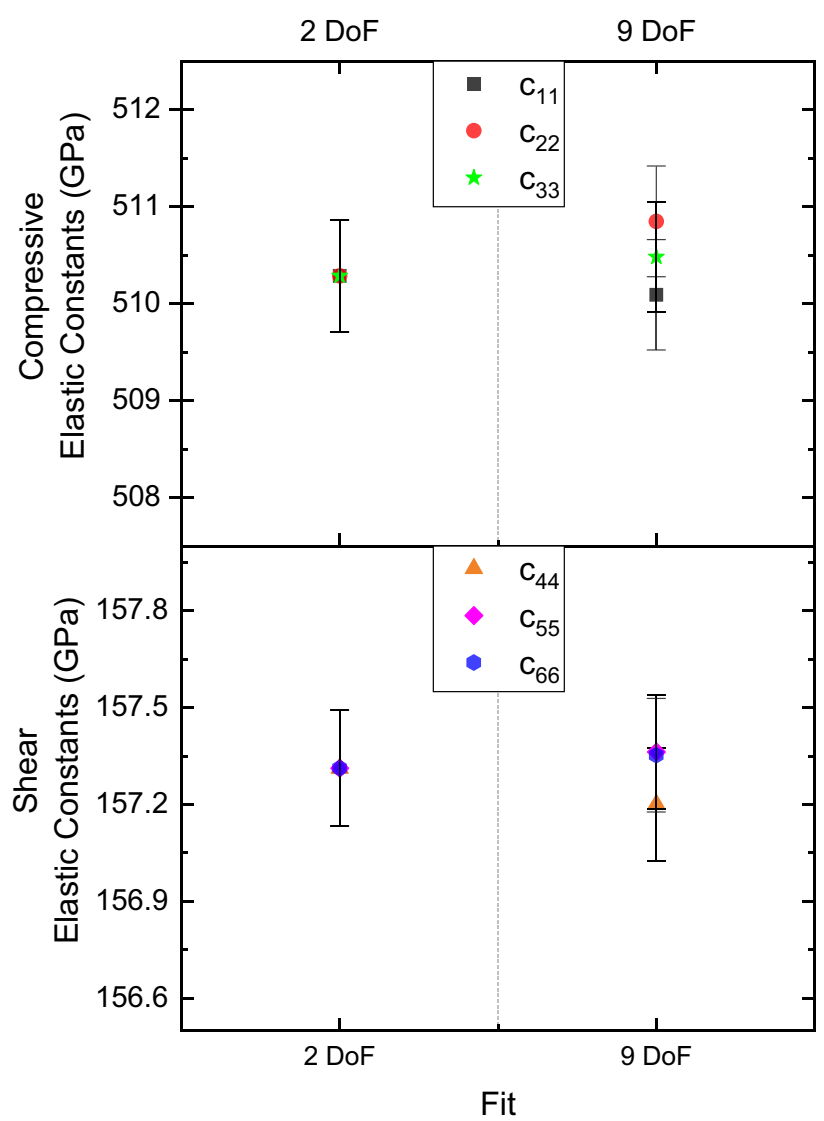

Figure 3 Compressive and shear elastic constants of a $99.95 \%$ pure tungsten RPR as determined by 2-DoF and 9-DoF fits using RUS.

RPRs, and a microstructure that is homogeneous with a correctly modeled symmetry [24]. The slight deviations in the 9-DoF elastic constants, as well as the slightly smaller errors for the $9=$ DoF fit in comparison with the 2-DoF fit, are attributed to the availability of more free parameters in the 9-DoF fit minimization scheme. Given that the 9-DoF fit is in such close agreement with the isotropic fit, this demonstrates that the isotropic assumption is correct for this material.

Figure 4 displays the results of fitting the RUS spectrum obtained from an AA-1100-O RPR using an isotropic fit (2 DoF) and a hexagonal symmetry (5 DoF) for different orientations. For 2-DoF, the RMS error is $0.85 \%$. This error is nearly ten times higher than for the isotropic fit of the tungsten RPR. The increase in the RMS for the 2-DoF fit of the AA-1100 is not due to lack of accuracy in the density or geometry of these samples, as they are very similar to those of the tungsten RPRs. 


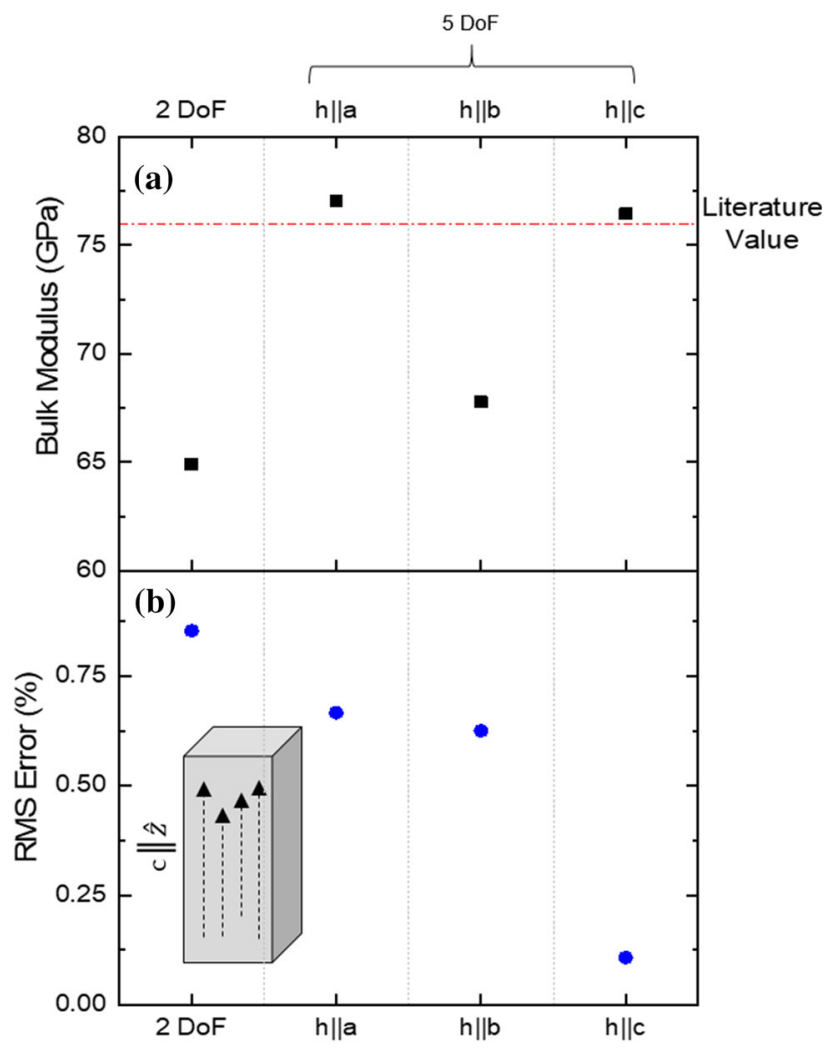

Symmetry and Texture Axis Orientation of the Fit

Figure 4 a Bulk modulus compared to literature value [78] and b RMS error using different symmetry and orientation fits for an AA-1100-O sample with $c \| \widehat{z}$.

The increase in RMS error indicates that the use of the isotropic fit to analyze the RUS spectra of extruded AA-1100-O is not correct. For these AA-1100 samples, the effects of extrusion-induced texture cannot be ignored and are a significant deviation from the assumption of the random polycrystalline orientation distribution. We also find a $16 \%$ deviation from the literature value for bulk modulus $B$ [79]. Thus, we analyze the same spectrum with a 5-DoF fit (see Fig. 4) for a sample machined with $c|| \widehat{z}$ (see Fig. 1b). The 5-DoF fit was then performed using different symmetry orientations, i.e., with $\boldsymbol{h}|| \boldsymbol{a}$, $\boldsymbol{h}|| \boldsymbol{b}$, and $\boldsymbol{h}|| \boldsymbol{c}$. Only the latter $(\boldsymbol{h}|| c$, i.e., $h|| \widehat{z})$ fit matches with the sample's symmetry. The RMS error decreases dramatically when the correct symmetry is chosen (see Fig. 4a). It is remarkable that although the RMS error of the 2-DoF fit is $0.85 \%$, the differences in the values obtained for $B$ are as large as $16 \%$ (see Fig. $4 \mathrm{~b}$ ). This highlights the importance of choosing the correct symmetry. When fitting with the correct symmetry, the values for $B$ are consistent with the values from the literature for polycrystalline aluminum [79]. The complete determination of the elastic constants $c_{i j}$ is shown in Table 1 for the 2- and 5 -DoF fits performed in the different orientations. It could also be argued that the lower RMS obtained with 5-DoF fits is an artifact of a higher number of fitting parameters used as compared to the 2-DoF fit. However, the results for $\boldsymbol{h} \| \boldsymbol{a}$ and $\boldsymbol{h} \| \boldsymbol{b}$ indicate otherwise.

There are cases when the true symmetry of the material is unknown a priori. The elastic constants can be extracted using a fitting procedure with an even lower symmetry, such as an orthorhombic fit (9 DoF) for which $\mathrm{c}_{11}, \mathrm{c}_{22}, \mathrm{c}_{33}, \mathrm{c}_{23}, \mathrm{c}_{13}, \mathrm{c}_{12}, \mathrm{c}_{44}, \mathrm{c}_{55}$ and $\mathrm{c}_{66}$ are independent. If the material truly has transversely isotropic texture, then the 9-DoF fit should yield identical results (within errors) to the 5-DoF fit. RMS error is expected to be slightly less for a 9-DoF fit because there are more free parameters available in the minimization scheme, analogously to the tungsten case. Figure 5 compares the results obtained using 5-DoF and 9-DoF fits for the shear constants $\left(c_{44}, c_{55}\right.$ and $\left.c_{66}\right)$ of an AA-1100-O sample with $c \| \widehat{z}$. The determined value of $\mathrm{c}_{66}$ is identical to the $5-\mathrm{DoF}$ and 9-DoF fits, and the values of $c_{44}$ and $c_{55}$ are within the error. More importantly, the results shown in Fig. 5 confirm the assumption of transverse isotropy for the extruded material, without the need of determining the material's symmetry or its orientation a priori. Note that the level of anisotropy in the shear constants is clearly outside the error of determination around $6 \%$.

RUS is capable of determining the degree of elastic anisotropy for samples that were machined regardless of the orientations with respect to the rod. The compressive, dilatational and shear elastic constants for samples machined in different orientations are shown in Fig. 6. Henceforth, all fits were performed using a 5-DoF fit with $\boldsymbol{h} \| \widehat{z}$. Although there are small variations in the elastic constants from sample to sample, this is likely due to handling and small differences in machining quality. The RMS errors of the fits are all low, about $0.1 \%$. The general relationships between the elastic constants are the same for each sample: $c_{11}>c_{33}, c_{13}>c_{12}$, and $c_{44}>c_{66}$. These samples have much larger anisotropy in the shear constants than in the compressive constants. Note that $c_{12}$ is set by the values of the other constants (see Eq. 9) since hexagonal symmetry is associated with 
Table 1 Elastic constants and RMS error for an AA-1100-O sample machined $c \| \widehat{z}$ as determined by fits with $h \| a$, $h \| b$ and $h \| c$ (best fit). All elastic constants/moduli are in GPA

\begin{tabular}{lllll}
\hline $\begin{array}{l}\text { Fit Parameter } \\
(\text { GPA })\end{array}$ & $\begin{array}{l}\text { 2-DoF } \\
\text { Fit }\end{array}$ & $\begin{array}{l}\text { 5-DoF Fit } \\
\text { Assuming } h \| a\end{array}$ & $\begin{array}{l}\text { 5-DoF Fit } \\
\text { Assuming } h \| b\end{array}$ & $\begin{array}{l}\text { 5-DoF Fit } \\
\text { Assuming } h \| c\end{array}$ \\
\hline$c_{11}$ & 100.0 & 109.4 & 99.8 & 111 \\
$c_{33}$ & 100.0 & 114.7 & 107.6 & 109 \\
$c_{13}$ & 47.4 & 61.6 & 53.1 & 59.6 \\
$c_{44}$ & 26.3 & 27 & 27.1 & 27.4 \\
$c_{66}$ & 26.3 & 26.1 & 26.7 & 25.9 \\
B & 64.9 & 77 & 67.8 & 76.4 \\
RMS error (\%) & 0.85 & 0.67 & 0.62 & 0.11 \\
\hline
\end{tabular}

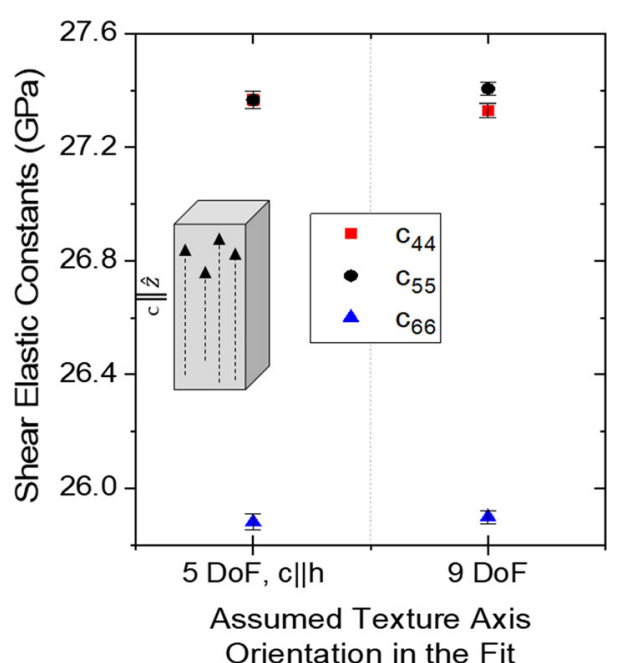

Figure 5 Comparison of 9-DoF fit vs. 5-DoF fit shear elastic constants with $\mathbf{c} \| \boldsymbol{h}$ using RUS for an AA-1100 sample machined with $\mathbf{c} \| \widehat{z}$.

five independent constants, but both dilatational constants are shown in Fig. 6 for comparison purposes.

In order to obtain more statistical confidence in the anisotropy inferred from the results shown above, thirteen additional AA-1100-O samples were characterized, which were machined in the same fashion from the same rod and with identical thermal histories as described before. The RUS spectra were collected for each of the RPRs and were used to perform hexagonal (5 DoF) fits with $h \| \widehat{z}$ for each of the samples. The elastic constants for these samples are displayed in Fig. 7, along with the anisotropy ratios of the compressive and shear elastic constants. The anisotropy in the shear elastic constants $(5.7 \% \pm 0.5 \%)$ is consistently larger than the anisotropy in dilatational elastic constants $(2.4 \% \pm 0.6 \%)$ and larger than the anisotropy in compressive elastic constants $(1.5 \% \pm 0.5 \%)$ as determined by RUS. These findings are in close agreement with the PE
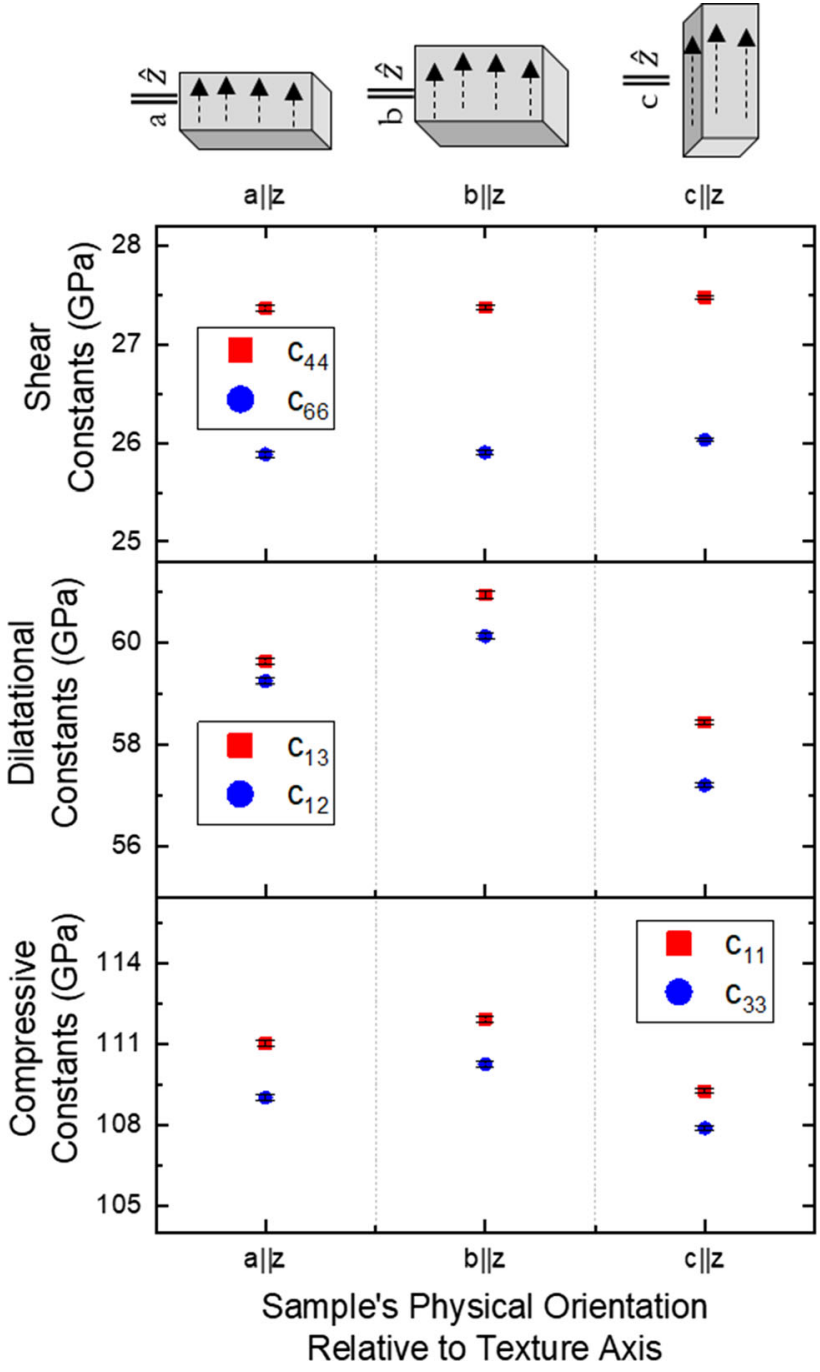

Figure 6 RUS determination of elastic constants of AA-1100-O RPRs vs. physical orientation relative to texture axis, where all fits were performed with $\boldsymbol{h} \| \widehat{z}$.

results performed on two samples ( 3 and 5) for validation purposes, particularly the anisotropy ratios. From Fig. $7 \mathrm{a}$, it can be seen that the $c_{i j}$ determined by pulse echo are slightly lower than those determined 
by the RUS technique. It is possible that this is due to the interaction of the propagating wave with the boundaries of the sample, which can guide and slow the wave, resulting in lower values of $c_{i j}$. We also see small variations in the elastic constants from sample to sample as expected due to different aspects such as sample inhomogeneity, handling and small differences in machining quality. The variation is relatively bigger for the compression constants as the anisotropy is smaller. The difference in density is one order of magnitude smaller than the variations observed in $c_{i j}$ values from sample to sample, thus not the origin of sample-to-sample variation found in $c_{i j}$. We did observe a non-systematic change in elastic constant obtained by RUS before and after sound velocity measurements, of the same order of the dispersion between samples. Nevertheless, the degree of anisotropy remains practically unchanged.

The determination of the degree of elastic anisotropy is paramount toward understanding the mechanical properties of the material. In this particular case, with extruded AA-1100-O, anisotropy in the shear elastic constants is nearly 6\% (see Fig. 7a). The engineering constants can be calculated from the elastic constants by using Eqs. 10-13. For these samples, the Young's moduli and Poisson ratios vary by $3 \%$ and $11 \%$, respectively.

Another common measure of anisotropy was proposed by Thomsen [80], given by these parameters:

$\varepsilon=\frac{c_{11}-c_{33}}{2 c_{33}}$

$\gamma=\frac{c_{66}-c_{44}}{2 c_{44}}$

$\delta=\frac{\left(c_{13}+c_{44}\right)^{2}-\left(c_{33}-c_{44}\right)^{2}}{2 c_{33}\left(c_{33}-c_{44}\right)}$

The first two anisotropy parameters $(\varepsilon$ and $\gamma)$ are the normalized difference of the compressive and shear elastic constants. The parameter $\delta$ reflects the near-vertical compression wave anisotropy and does not include $c_{11}$ (the horizontal velocity) [80]. For weak anisotropy, $\delta$ can be further reduced to $\delta_{\text {weak }}=\left(\mathrm{c}_{13^{-}}\right.$ $\left.\left(c_{33}-2 c_{44}\right)\right) / c_{33}$, so $\delta$ can also be seen as the off-diagonal or dilatational constants anisotropy factor. Interestingly, when the $\delta$ is plotted against $\varepsilon$ and $\gamma$, we find a clear correlation between them with $\delta=0.036+2.88 \varepsilon$ and $\delta=-0.01+2.17 \gamma$ in Fig. $7 \mathrm{~b}$. This suggests that the differences in $c_{i j}$ between samples are not due to random variation or error in their determination but in different levels of anisotropy among the samples.

To further investigate the origin of the elastic anisotropy, we performed neutron measurements to determine the texture and calculated the elastic anisotropy using self-consistent model using those neutron textures.

Figure 8 shows the resulting pole figures and illustrations of the samples machined with the extrusion direction parallel to the $c$ (Fig. 8a and b) and $a$ (Fig. $8 \mathrm{c}$ and $\mathrm{d}$ ). As shown in the pole figures, both samples have similar MRD values, indicating that, other than the rotation, texture is identical for the two samples. However, the plane of isotropy for the sample shown in Fig. 8a is clearly the $a-b$ plane, while the plane of isotropy for the sample shown in Fig. $8 c$ is the $b-c$ plane, both of which are consistent with RUS and PE results. It is worth noting that there are very small deviations from transverse isotropy; e.g., the light blue "ring" in the top 111 pole figure is not of constant density around the ring, and the vertical band in the bottom 200 pole figure is not vertical.

As mentioned in "Neutron diffraction" section, the measured ODFs of samples $\mathbf{c} \| \widehat{z}$ and $\mathbf{a} \| \widehat{z}$ were integrated in small regions of orientation space to give a set of 23,328 orientations. These sets of individual orientations representing the textures of samples $\mathbf{c} \| \widehat{z}$ and $\mathbf{a} \| \widehat{z}$ were used as input of ELSC calculations. The other adopted input parameters were the values of $\mathrm{Al}$ single-crystal elastic constants $c_{11}^{A l-S X}=107 \mathrm{GPa}, \quad c_{12}^{A l-S X}=61 \mathrm{GPa} \quad$ and $\quad c_{44}^{A l-S X}=$ $28 \mathrm{GPa}$ obtained by averaging five different measurements at room temperature reported in [78], and a grain's ellipsoidal shape (1:1:5) along $x, y$ and $z$, consistent with the morphology of grains extruded in $\widehat{z}$. (Note that the reported results are fairly insensitive to the actual ratio between the long axis and the short axes of the ellipsoid.)

Figure 9 shows the ELSC predictions of nine polycrystal's elastic stiffness components: $c_{11}, c_{22}, c_{33} ; c_{12}, c_{13}, c_{23}$ and $c_{44}, c_{55}, c_{66}$ for samples c $\| \widehat{z}$ and $\mathbf{a} \| \widehat{z}$. Note that symmetry of $c_{i j k l}$ was not imposed but arose naturally from the symmetry of the input textures. It is observed that: a) the predicted polycrystal elastic constants are very close to transversely isotropic symmetry (or "axial symmetry" or "hexagonal symmetry"). For example, in the case of c $\| \widehat{z}$ sample, $c_{11} \cong c_{22}, c_{13} \cong c_{13}, c_{44} \cong c_{55}$ and $c_{12} \cong$ 

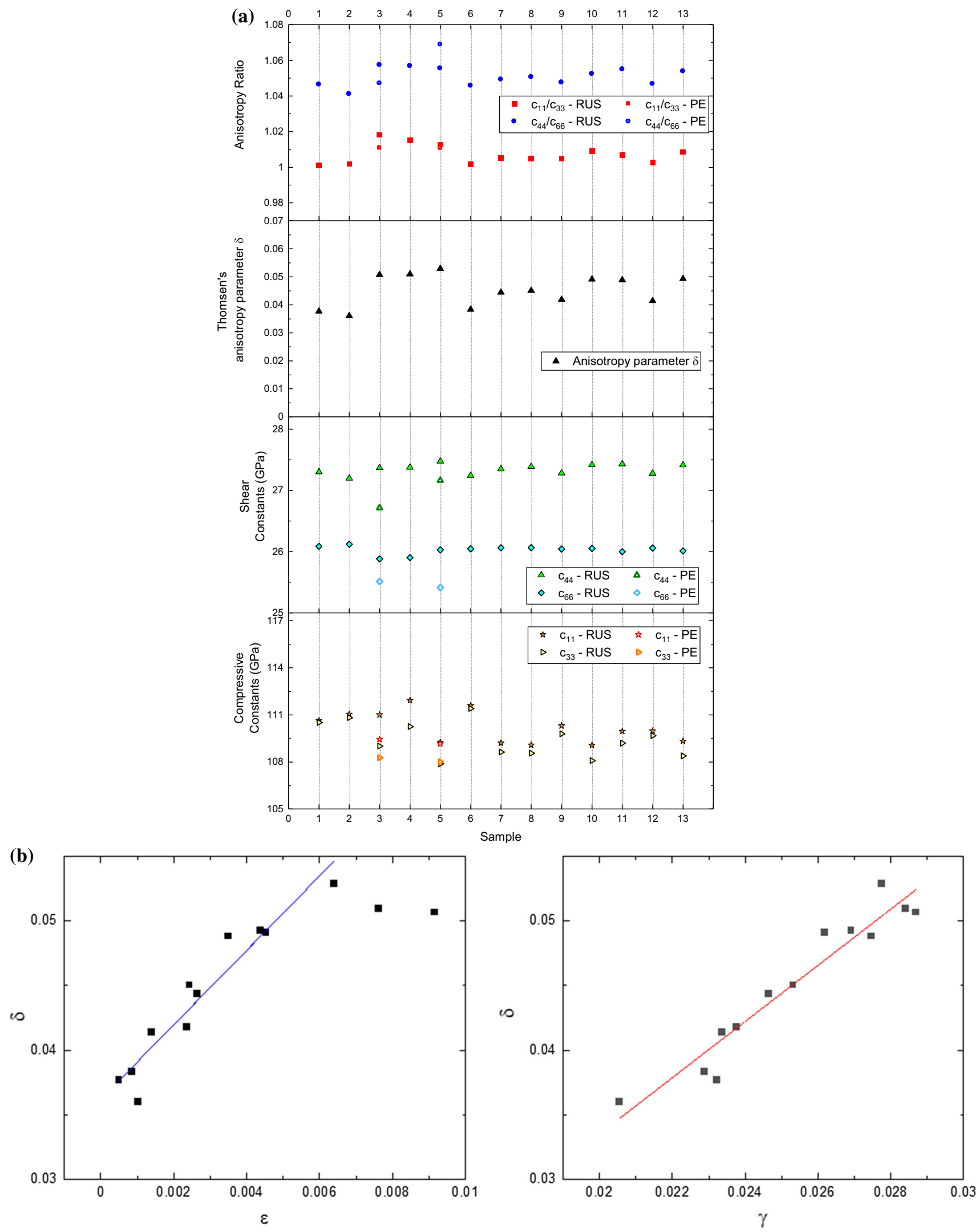
4 Figure 7 a Elastic constants and anisotropy ratios of several AA1100 RPRs as determined by RUS and PE. Error bars are the same size as the symbols, $\mathbf{b}$ Thomsen's anisotropy parameter $\delta$ as a function of $\varepsilon$ and $\gamma$.

$c_{11}-2 c_{66}$ within a tolerance better than $0.1 \%$; b) the difference between the $\mathbf{c} \| \widehat{z}$ and $\mathbf{a} \| \widehat{z}$ is essentially a switch between the sample directions, $\mathrm{z}$ and $\mathrm{x}$, with $\mathrm{x}$ becoming the direction of the symmetry axis, and consequently $\quad c_{11} \neq c_{22} \cong c_{33}, \quad c_{23} \neq c_{12} \cong c_{13}, \quad c_{44} \neq$ $c_{55} \cong c_{66}$ and $c_{23} \cong c_{33}-2 c_{44}$. These results, obtained from textures of samples cut from the same rod but independently measured, show consistency between the texture measurements and modeling methodology.

Figure 10 shows the comparisons between the polycrystal's elastic constants measured with RUS (a)
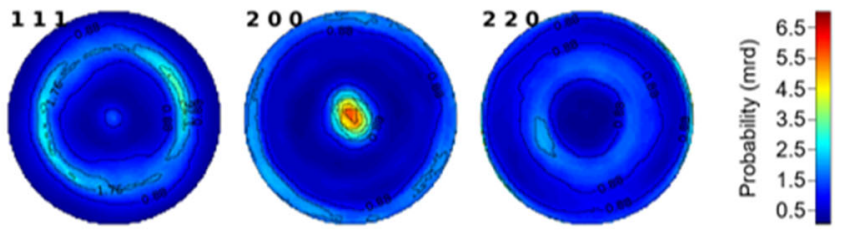

(c)
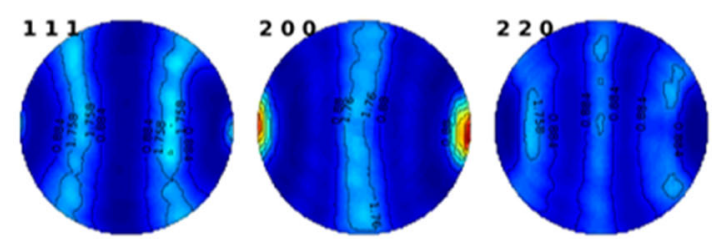

(b)

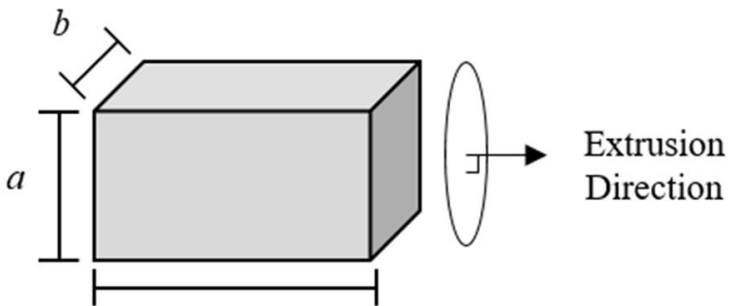

(d)

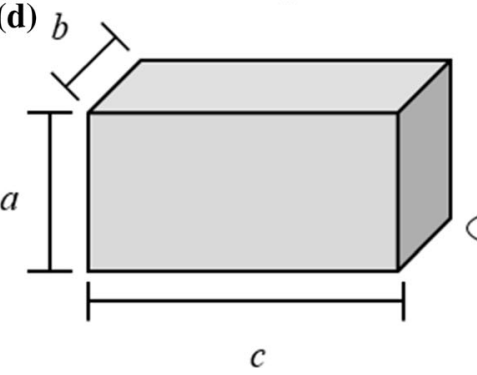

Extrusion

Direction

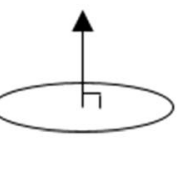

Figure 8 a Neutron diffraction pole figures for an aluminum 1100 RPR whose extrusion direction is parallel to " $c$ " (c $\| \widehat{z})$ as illustrated in (b), and c neutron diffraction pole figures for an aluminum 1100 RPR whose extrusion direction is parallel to " $a$ ” (a) $\| \widehat{z})$ as illustrated in $(\mathbf{d})$.

Figure 9 ELSC predictions of polycrystal's elastic constants with input from ND textures for samples $\mathbf{c} \| \widehat{z}$ and $\mathbf{a} \| \widehat{z}$.

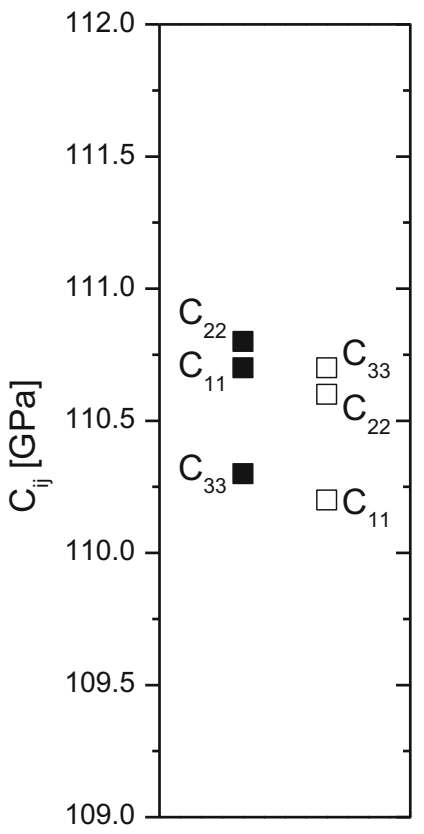

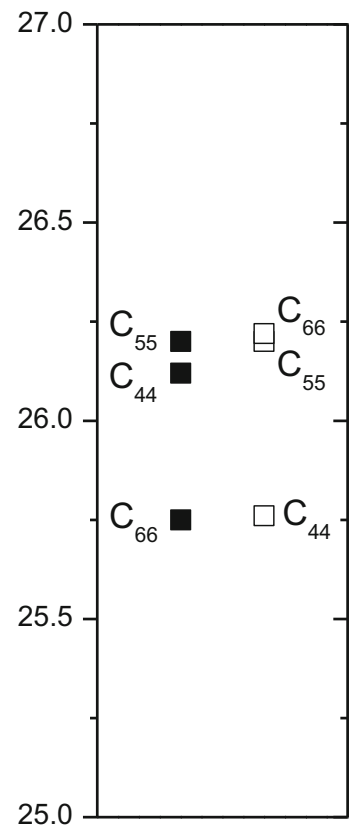


Figure 10 a Elastic constants for a polycrystalline sample c $\| \widehat{z}$ measured with RUS and predicted with ELSC with input from ND textures. $\mathbf{b}$ Elastic constants for a polycrystalline sample a $\| \widehat{z}$ measured with RUS and predicted with ELSC with input from ND textures. (a)

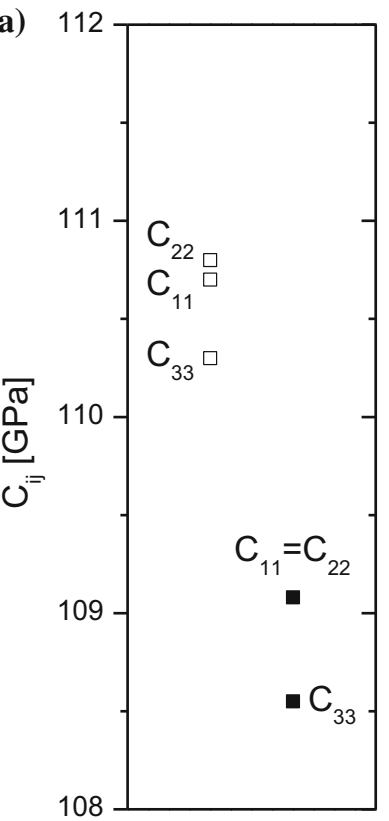

(b)

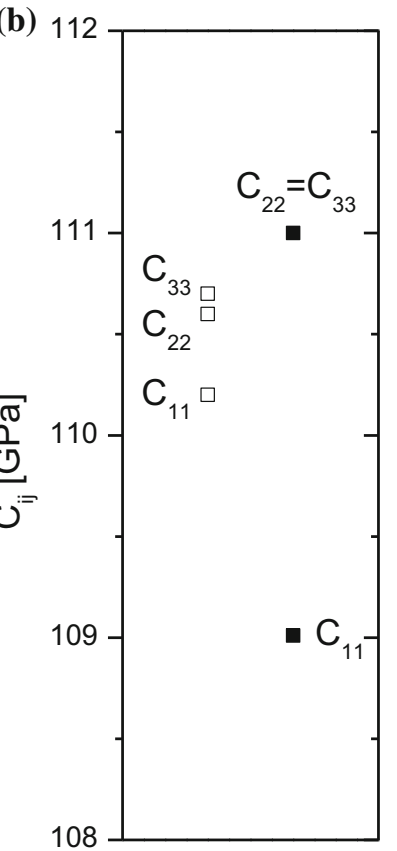

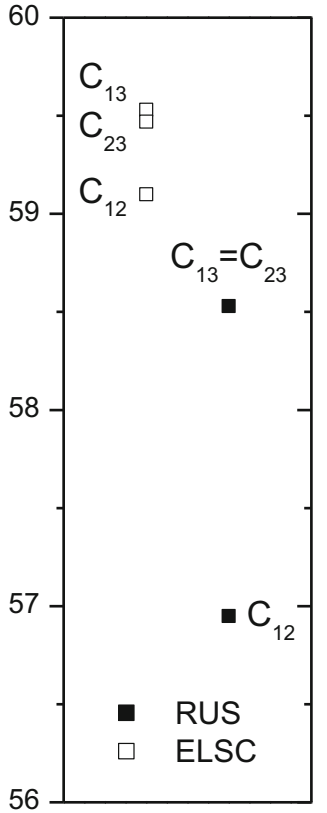
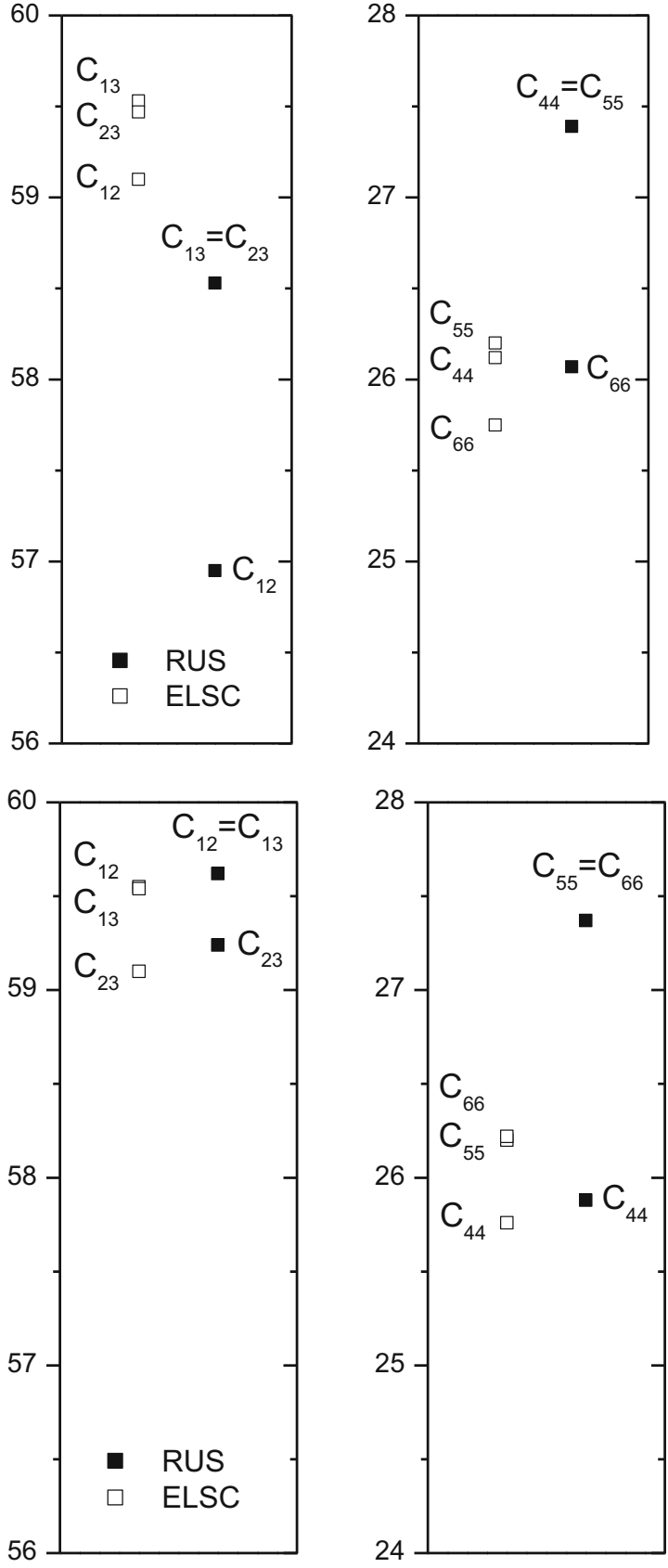

and predicted with ELSC with input from ND textures for samples $\mathbf{c} \| \widehat{z}$ and $\mathbf{a} \| \widehat{z}$, respectively. Note that ranges of all vertical axes are $4 \mathrm{GPa}$, for a fair visual comparison of the results. It is observed that the predicted anisotropy is qualitatively consistent with the RUS measurements, e.g., in the case of sample $\quad$ cl| $\widehat{z}: \quad c_{33}<c_{11} \cong c_{22}, \quad c_{12}<c_{13} \cong c_{23}$, and $c_{66}<c_{44} \cong c_{55}$, although quantitative, the predictions and measurement are off, in some of the worst cases, by several percent. The quantitative differences between calculation and experiments may be due to systematic errors in the texture and intrinsic to the RUS analysis, namely, the measured texture by neutrons is not perfectly transverse isotropic as assumed (see Fig. 8), the single crystal elastic constants of AA$1100-\mathrm{O}$ are not the ones found in a single crystal extracted from tables, and/or model shortcomings. Elucidation of the ultimate causes of quantitative disagreement between experiments and predictions is currently the subject of investigation, including the 
study of materials with higher intrinsic single-crystal anisotropy.

As mentioned before, aluminum has a relatively low Zener anisotropy factor of $A=1.22$, meaning that its elastic properties are less sensitive to anisotropic changes due to texture than, say copper $(A=3.21)$, or 304 stainless steel $(A=3.77)$ [44] and many other common materials with larger $A$ factors, for which the degree of anisotropy due to texture will be much larger. Indeed, using the same texture measured for AA-1100, we calculated the polycrystal elastic constants that would result from $\mathrm{Cu}$ and SS samples with identical texture, as shown in Fig. 11, using a normalization based on the corresponding isotropic elastic constants in the case of random texture, for each material. Clearly, the same texture has larger effect on the elastic anisotropy as the $A$ increases. The changes in compression constants are smaller than those found in shear. We find an overall decrease in the average of the compression constant with higher $A$. For shear, the anisotropy is larger and there is a decrease in $\mathrm{c}_{66}$ clearly seen for $\mathrm{Cu}$ and SS.

\section{Summary and conclusions}

In this study, we use resonant ultrasound spectroscopy (RUS) to nondestructively determine the entire elastic tensor in extruded AA-1100-O (commercially pure aluminum in its annealed state with

Figure 11 Normalized elastic constants (vs. randomly distributed elastic constant) of AA-1100, $\mathrm{Cu}$ and SS using the same experimental texture.

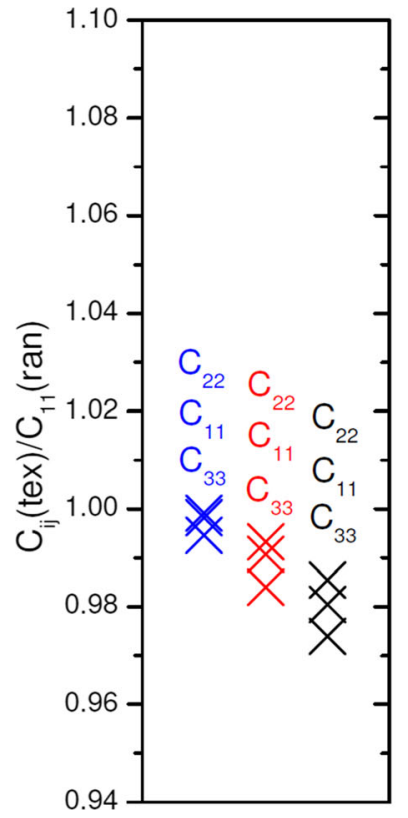

no cold working), which has transversely isotropic symmetry (five independent elastic constants) due to the texture induced by the extrusion process. The relative anisotropy of the compressive ( $c_{11}$ vs. $\left.c_{33}\right)$ and shear ( $\mathrm{c}_{44}$ vs. $\mathrm{c}_{66}$ ) elastic constants is $1.5 \% \pm 0.5 \%$ and $5.7 \% \pm 0.5 \%$, respectively, where $c_{33}$ and $c_{66}$ are the elastic constants associated with the axis of symmetry of the texture. Because aluminum is an inherently low-anisotropy material whose Zener anisotropy factor is 1.22 , other common alloys (steels, nickel-based superalloys, copper-based alloys, etc.) are expected to have larger anisotropic properties given the same texture.

The necessity of accounting for the anisotropy resulting from the extrusion process has been demonstrated by comparing the RUS results from AA-1100-O samples fit using the isotropic assumption (2 DoF), the transverse isotropic (5 DoF) and orthotropic (9 DoF) fits. The error associated with describing extruded aluminum with an isotropic fit is supported by the extremely low-error, high-accuracy RUS results of the isotropic fit in $99.95 \%$ pure tungsten, which has a Zener anisotropy factor of unity and is therefore isotropic regardless of texture. The ability for RUS to determine the orientation of the texture axis has also been demonstrated by analyzing the results with a lower symmetry (9 DoF) and changing the orientation of the fit with respect of extrusion orientation (5 DoF). The elastic constants and the degree of elastic anisotropy as determined

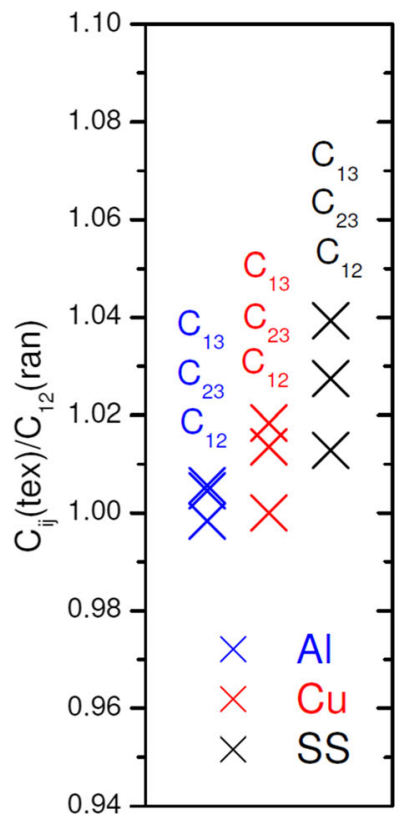


using RUS have been confirmed by direct sound velocity measurements using the ultrasonic pulseecho technique. The elastic constants were then used to calculate the engineering constants, whereby the Young's moduli and Poisson ratios parallel vs. perpendicular to the extrusion direction in these samples differ by $3 \%$ and $11 \%$, respectively. Using the experimental texture obtained by neutron diffraction, elastic self-consistent micromechanical simulations show good agreement in the level of anisotropy and small disagreement in the absolute values that we assign to material changes produced by handling the soft-annealed AA-1000 samples. Using the same texture and calculation method, we show that the texture will have much larger effect for materials with larger Zener parameter, resulting even in lowering of the elastic constants.

\section{Acknowledgements}

Research presented in this article was supported by the Laboratory Directed Research and Development program of Los Alamos National Laboratory under project 20180250ER. Work at the National High Magnetic Field Laboratory at LANL, was also supported by the National Science Foundation through NSF/DMR-1644779 and the state of Florida (F.F.B., J.B.). Work by B.T.S. was supported by LANL Office of Experimental Science Dynamic Materials Properties program (Campaign 2). The work has benefitted from the use of the Los Alamos Neutron Science Center (LANSCE) at LANL. Los Alamos National Laboratory is operated by Triad National Security, LLC, for the National Nuclear Security Administration of the U.S. Department of Energy under contract number 89233218NCA000001.

\section{Compliance with ethical standards}

Conflict of interest The authors declare no competing interest.

Open Access This article is licensed under a Creative Commons Attribution 4.0 International License, which permits use, sharing, adaptation, distribution and reproduction in any medium or format, as long as you give appropriate credit to the original author(s) and the source, provide a link to the Creative Commons licence, and indicate if changes were made. The images or other third party material in this article are included in the article's Creative Commons licence, unless indicated otherwise in a credit line to the material. If material is not included in the article's Creative Commons licence and your intended use is not permitted by statutory regulation or exceeds the permitted use, you will need to obtain permission directly from the copyright holder. To view a copy of this licence, visit http://creativecommons.org/licen ses $/$ by $/ 4.0 /$.

\section{Appendix}

The relationship between the phase velocities of ultrasonic plane waves and the elastic constants of the material can be determined by solving the eigenvalue problem associated with the Christoffel equation in Eq. 18, where $\rho$ is the density, $v$ is the elastic wave phase velocity, $\delta_{i j}$ is the Kronecker delta, $\Gamma_{i j}=c_{i j k l} n_{j} n_{l}$ is the Christoffel tensor, and $n_{j}$ are the directional cosines of the elastic wave propagation direction for a given reference axis [81-83]. For isotropic materials (two independent elastic constants, see Eq. 3 where $c_{12}=c_{11}-2 c_{44}$ ), the Christoffel tensor reduces to the relations in Eq. 19:

$$
\begin{aligned}
&\left|\Gamma_{i j}-\rho v^{2} \delta_{i j}\right|=0 \\
& \Gamma_{i j}=\left|\begin{array}{lll}
\Gamma_{11} & \Gamma_{12} & \Gamma_{13} \\
\Gamma_{21} & \Gamma_{22} & \Gamma_{23} \\
\Gamma_{31} & \Gamma_{32} & \Gamma_{33}
\end{array}\right| \\
& \Gamma_{11}=c_{11} n_{1}^{2}+c_{44} n_{2}^{2}+c_{44} n_{3}^{2} \\
& \Gamma_{22}=c_{44} n_{1}^{2}+c_{11} n_{2}^{2}+c_{44} n_{3}^{2} \\
& \Gamma_{33}=c_{44} n_{1}^{2}+c_{44} n_{2}^{2}+c_{11} n_{3}^{2} \\
& \Gamma_{12}=n_{1} n_{2}\left(c_{12}+c_{44}\right) \\
& \Gamma_{23}=n_{2} n_{3}\left(c_{12}+c_{44}\right) \\
& \Gamma_{13}=n_{1} n_{3}\left(c_{12}+c_{44}\right)
\end{aligned}
$$

Since changing the orientation in which the elastic wave velocity measurement is performed in an isotropic material yields identical results, we arbitrarily assign the $z$-axis as the direction of elastic wave propagation, i.e., $n_{x}=n_{y}=0$ and $n_{z}=1$. It follows that:

$$
\left[\begin{array}{ccc}
c_{44}-\rho v^{2} & 0 & 0 \\
0 & c_{44}-\rho v^{2} & 0 \\
0 & 0 & c_{11}-\rho v^{2}
\end{array}\right]\left[\begin{array}{l}
u_{x} \\
u_{y} \\
u_{z}
\end{array}\right]=\left[\begin{array}{l}
0 \\
0 \\
0
\end{array}\right]
$$

and 
$\left(c_{44}-\rho v^{2}\right)^{2}\left(c_{11}-\rho v^{2}\right)=0$

where $u_{i}$ is the polarization vector which represents the atomic displacement directions. The roots of the above relationship are the eigenvalues of the Christoffel tensor which relate the transverse $\left(v_{S}\right)$ and longitudinal $\left(v_{L}\right)$ phase velocities to the elastic constants of an isotropic solid, as shown in Eq. 22:

$$
\begin{aligned}
& {\left[\begin{array}{ccc}
c_{11} \sin ^{2} \theta+c_{66} \cos ^{2} \theta-\rho v^{2} & \left(c_{12}+c_{66}\right) \sin \theta \cos \theta & 0 \\
\left(c_{12}+c_{66}\right) \sin \theta \cos \theta & c_{66} \sin ^{2} \theta+c_{11} \cos ^{2} \theta-\rho v^{2} & 0 \\
0 & 0 & c_{44}-\rho v^{2}
\end{array}\right]} \\
& {\left[\begin{array}{l}
u_{x} \\
u_{y} \\
u_{z}
\end{array}\right]=\left[\begin{array}{l}
0 \\
0 \\
0
\end{array}\right]}
\end{aligned}
$$

$c_{44}=\rho v_{T}^{2}$
$c_{11}=\rho v_{L}^{2}$

Note that because there is no orientation dependence in isotropic materials, the entire elastic tensor can be determined from a single pulse-echo experiment. For transversely isotropic materials (five independent elastic constants, see Eq. 9), however, the Christoffel tensor is described by the relations in Eq. 23. In this analysis, the plane of isotropy has been assigned to the $\mathrm{x}-\mathrm{y}$ plane, and the " $\mathrm{z}$ " direction is parallel to the textured axis.

$\Gamma_{11}=c_{11} n_{1}^{2}+c_{66} n_{2}^{2}+c_{44} n_{3}^{2}$

$\Gamma_{22}=c_{66} n_{1}^{2}+c_{11} n_{2}^{2}+c_{44} n_{3}^{2}$

$\Gamma_{33}=c_{44} n_{1}^{2}+c_{44} n_{2}^{2}+c_{33} n_{3}^{2}$

$\Gamma_{12}=n_{1} n_{2}\left(c_{12}+c_{66}\right)$

$\Gamma_{23}=n_{2} n_{3}\left(c_{13}+c_{44}\right)$

$\Gamma_{13}=n_{1} n_{3}\left(c_{13}+c_{44}\right)$

Under these symmetry conditions, the direction of wave propagation is now relevant. Following the same methodology as before, we now consider a plane wave propagating in the $x-y$ plane in a direction $\theta$ with respect to the z-axis. Therefore, $n_{1}=\sin \theta$, $n_{2}=\cos \theta, \quad n_{3}=0$, and the Christoffel equation becomes Eq. 24. Similarly, for a plane wave propagating in the $\mathrm{x}-\mathrm{z}$ plane in a direction $\theta$ with respect to the z-axis, $n_{1}=\sin \theta, n_{2}=0, n_{3}=\cos \theta$, and the Christoffel equation becomes Eq. 25.
The ultrasonic phase velocities relate to the elastic constants of a transversely isotropic material by Eq. 26, where $v_{T, q}, v_{T}$ and $v_{L}$ are the quasi-transverse, pure transverse and longitudinal velocities, and $\theta$ is the angle between the propagation direction and the z-axis of symmetry (i.e., $\theta=0^{\circ}$ for waves propagating along the z-axis).

$$
\begin{aligned}
v_{L}= & \sqrt{\frac{c_{11} \sin ^{2} \theta+c_{33} \cos ^{2} \theta+c_{44}+\sqrt{M}}{2 \rho}} \\
v_{T, q}= & \sqrt{\frac{c_{11} \sin ^{2} \theta+c_{33} \cos ^{2} \theta+c_{44}-\sqrt{M}}{2 \rho}} \\
v_{T}= & \sqrt{\frac{c_{66} \sin ^{2} \theta+c_{44} \cos ^{2} \theta}{\rho}} \\
M= & {\left[\left(c_{11}-c_{44}\right) \sin ^{2} \theta+\left(c_{33}-c_{44}\right) \cos ^{2} \theta\right]^{2} } \\
& +\left(c_{13}+c_{44}\right)^{2} \sin ^{2} 2 \theta
\end{aligned}
$$

The relationship between sound velocities and sample orientations for $c_{13}$ is given in Eq. 27 for an experiment performed at $45^{\circ}$ from the texture axis. Samples were not machined in such off-angle orientations in this work; therefore, pulse echo was unable to obtain information related to the values of $c_{13}$ for the AA-1100 samples in this study. 


$$
\begin{aligned}
& c_{11}=\left.\rho v_{L}^{2}\right|_{\theta=90^{\circ}} \\
& c_{33}=\left.\rho v_{L}^{2}\right|_{\theta=0^{\circ}} \\
& c_{12}=c_{11}-2 c_{66} \\
& c_{13}=\sqrt{\left.4 \rho^{2} v_{L}^{4}\right|_{\theta=45^{\circ}}-\left.2 \rho v_{L}^{2}\right|_{\theta=45^{\circ}}\left(c_{11}+c_{33}+2 c_{44}\right)+\left(c_{11}+c_{44}\right)\left(c_{33}+c_{44}\right)}-c_{44} \\
& c_{44}=\left.\rho v_{T}^{2}\right|_{\theta=0^{\circ}} \\
& c_{66}=\left.\rho v_{T}^{2}\right|_{\theta=90^{\circ}}
\end{aligned}
$$

The uncertainties associated with the pulse-echo experiments are calculated by the propagation of errors as described by the relationship shown in Eq. 28, where $\delta c_{i j}$ is the uncertainty of the elastic constant, $\delta \rho$ is the uncertainty of density, and $\delta v$ is the uncertainty of the measured sound velocity.

$\delta c_{i j}=c_{i j} \sqrt{\left(\frac{\delta \rho}{\rho}\right)^{2}+2\left(\frac{\delta v}{v}\right)^{2}}$.

\section{References}

[1] Hutchinson B (2015) Critical assessment 16: anisotropy in metals. Mater Sci Technol 31(12):1393-1401. https://doi.or g/10.1179/1743284715y.0000000118

[2] Ledbetter HM, Reed RP (1973) Elastic properties of metals and alloys, I. iron, nickel, and iron-nickel alloys. J Phys Chem Ref Data 2(3):531-618. https://doi.org/10.1063/1. 3253127

[3] Wang Y, Huang JC (2003) Texture analysis in hexagonal materials. Mater Chem Phys 81(1):11-26. https://doi.org/10. 1016/S0254-0584(03)00168-8

[4] Wenk HR, Van Houtte P (2004) Texture and anisotropy. Rep Prog Phys 67(8):1367-1428. https://doi.org/10.1088/0034-4 $885 / 67 / 8 / \mathrm{r} 02$

[5] Wenk HR (2002) Texture and anisotropy. Rev Mineral Geochem 51:291-329. https://doi.org/10.2138/gsrmg.51.1. 291

[6] Tang D, Fang WL, Fan XH, Zou TX, Li ZH, Wang HM, Li DY, Peng YH, Wu PD (2019) Evolution of the material microstructures and mechanical properties of AA1100 aluminum alloy within a complex porthole die during extrusion. Materials 12(1):14. https://doi.org/10.3390/ma12010016

[7] Hirosawa E (1964) Fiber textures of extruded aluminum alloy rod. T Jpn I Met 5(4):235-237. https://doi.org/10.2320/ matertrans 1960.5.235

[8] Zhang K, Marthinsen K, Holmedal B, Aukrust T, Segatori A (2018) Through thickness variations of deformation texture in round profile extrusions of 6063-type aluminium alloy: experiments, FEM and crystal plasticity modelling. Mater
Sci Eng A-Struct Mater Prop Microstruct Process 722:20-29. https://doi.org/10.1016/j.msea.2018.02.081

[9] Chen JA, Yan W, Li W, Miao JA, Fan XH (2011) Texture evolution and its simulation of cold drawing copper wires produced by continuous casting. T Nonferr Metal Soc 21(1):152-158. https://doi.org/10.1016/S1003-6326(11)606 $92-4$

[10] Mathur KK, Dawson PR (1990) Texture development during wire drawing. J Eng Mater-T Asme 112(3):292-297. http s://doi.org/10.1115/1.2903326

[11] Gan WM, Huang YD, Wang R, Zhong ZY, Hort N, Kainer KU, Schell N, Brokmeier HG, Schreyer A (2013) Bulk and local textures of pure magnesium processed by rotary swaging. J Magnes Alloy 1(4):341-345. https://doi.org/10. 1016/j.jma.2013.12.004

[12] Wronski M, Wierzbanowski K, Wronski S, Bacroix B (2015) Microstructure and texture of asymmetrically rolled aluminium and titanium after deformation and recrystallization. Iop Conf Ser-Mat Sci. https://doi.org/10.1088/1757-899x/8 9/1/012050

[13] Warren BE (1941) X-ray diffraction methods. J Appl Phys 12(5):375-384. https://doi.org/10.1063/1.1712915

[14] Randle V (2009) Applications of electron backscatter diffraction to materials science: status in 2009. J Mater Sci 44(16):4211-4218. https://doi.org/10.1007/s10853-009-357 $0-0$

[15] Brokmeier HG (1997) Neutron diffraction texture analysis. Phys B 234:977-979. https://doi.org/10.1016/S0921-4526(9 6)01230-6

[16] Brokmeier HG (1994) Neutron-diffraction texture analysis for industrial applications. Z Metallk 85(8):598-602

[17] Brokmeier HG (1994) Texture analysis by neutron-diffraction. Mater Sci Forum 157:59-70. https://doi.org/10.4028/ www.scientific.net/MSF.157-162.59

[18] Wenk HR (1991) Standard project for pole-figure determination by neutron-diffraction. J Appl Crystallogr 24:920-927. https://doi.org/10.1107/s0021889891004661

[19] Kim KY, Sachse W, Every AG (1993) On the determination of sound speeds in cubic-crystals and isotropic media using a broad-band ultrasonic point-source point-receiver method. J Acoust Soc Am 93(3):1393-1406. https://doi.org/10.1121/ 1.405426

[20] Espinoza C, Feliu D, Aguilar C, Espinoza-Gonzalez R, Lund F, Salinas V, Mujica N (2018) Linear versus nonlinear acoustic probing of plasticity in metals: a quantitative assessment. Materials. https://doi.org/10.3390/ma11112217

[21] Salinas V, Aguilar C, Espinoza-Gonzalez R, Lund F, Mujica $N$ (2017) In situ monitoring of dislocation proliferation during plastic deformation using ultrasound. Int $\mathrm{J}$ Plast 97:178-193. https://doi.org/10.1016/j.ijplas.2017.06.001 
[22] Migliori A, Sarrao JL (1997) Resonant ultrasound spectroscopy, applications to physics, materials measurements, and nondestructive evaluation. Wiley, New York

[23] Migliori A, Maynard JD (2005) Implementation of a modern resonant ultrasound spectroscopy system for the measurement of the elastic moduli of small solid specimens. Rev Sci Instrum 76(12):7. https://doi.org/10.1063/1.2140494

[24] Migliori A, Sarrao JL, Visscher WM, Bell TM, Lei M, Fisk Z, Leisure RG (1993) Resonant ultrasound spectroscopic techniques for measurement of the elastic-moduli of solids. Phys B 183(1-2):1-24. https://doi.org/10.1016/09214526(93)90048-b

[25] Levenberg K (1944) A method for the solution of certain non-linear problems in least squares. Q Appl Math 2(2):164-168

[26] Marquardt DW (1963) An algorithm for least-squares estimation of nonlinear parameters. J Soc Ind Appl Math 11(2):431-441. https://doi.org/10.1137/0111030

[27] Visscher WM, Migliori A, Bell TM, Reinert RA (1991) On the normal-modes of free-vibration of inhomogeneous and anisotropic elastic objects. J Acoust Soc Am 90(4):2154-2162. https://doi.org/10.1121/1.401643

[28] Lan B, Carpenter MA, Gan WM, Hofmann M, Dunne FPE, Lowe MJS (2018) Rapid measurement of volumetric texture using resonant ultrasound spectroscopy. Scr Mater 157:44-48. https://doi.org/10.1016/j.scriptamat.2018.07.029

[29] Lan B, Ben Britton T, Jun TS, Gan WM, Hofmann M, Dunne FPE, Lowe MJS (2018) Direct volumetric measurement of crystallographic texture using acoustic waves. Acta Mater 159:384-394. https://doi.org/10.1016/j.actamat.2018. 08.037

[30] Foster K, Fairburn SL, Leisure RG, Kim S, Balzar D, Alers G, Ledbetter H (1999) Acoustic study of texture in polycrystalline brass. J Acoust Soc Am 105(5):2663-2668. h ttps://doi.org/10.1121/1.426882

[31] Eros S, Reitz JR (1958) Elastic constants by the ultrasonic pulse echo method. J Appl Phys 29(4):683-686. https://doi. org/10.1063/1.1723250

[32] Sotnikov A, Schmidt H, Weihnacht M, Zhang SJ, Shrout TR, Yu FP (2012) Elastic constants of $\mathrm{YCa} 4 \mathrm{O}(\mathrm{BO} 3)(3)$ and $\mathrm{NdCa} 4 \mathrm{O}(\mathrm{BO} 3)(3)$ single crystals by the pulse-echo ultrasonic method. Ieee Int Ultra Sym. https://doi.org/10.1109/ Ultsym.2012.0623

[33] Hu EY, Wang WJ (2016) The elastic constants measurement of metal alloy by using ultrasonic nondestructive method at different temperature. Math Probl Eng. https://doi.org/10.11 $55 / 2016 / 6762076$

[34] Huntington HB (1947) Ultrasonic measurements on single crystals. Phys Rev 72(4):321-331
[35] Mcskimin HJ (1961) Pulse superposition method for measuring ultrasonic wave velocities in solids. J Acoust Soc Am 33(1):12-000

[36] Voigt W (1889) Ueber die Beziehung zwischen den beiden Elasticitätsconstanten isotroper Körper. Ann Phys 274(12):573-587

[37] Reuss A (1929) Berechnung der Fließgrenze von Mischkristallen auf Grund der Plastizitätsbedingung für Einkristalle. Zeitschrift für Angewandte Mathematik und Mechanik 9(1):49-58

[38] Hashin Z, Shtrikman S (1962) A variational approach to the theory of the elastic behaviour if polycrystals. J Mech Phys Solids 10(4):343-352. https://doi.org/10.1016/0022-5096(6 2) $90005-4$

[39] Hashin Z, Shtrikman S (1963) A variational approach to the theory of the elastic behaviour of multiphase materials. J Mech Phys Solids 11(2):127-140. https://doi.org/10.1016/ 0022-5096(63)90060-7

[40] Hershey AV (1954) The elasticity of an isotropic aggregate of the anisotropic cubic crystals. J Appl Mech-Trans ASME 21(3):236-240

[41] Lan B, Lowe MJS, Dunne FPE (2015) A spherical harmonic approach for the determination of HCP texture from ultrasound: a solution to the inverse problem. J Mech Phys Solids 83:179-198. https://doi.org/10.1016/j.jmps.2015.06.014

[42] Zener C (1948) Elasticity and anelasticity of metals. University of Chicago Press, Chicago, Illinois

[43] Thomas JF (1968) Third-order elastic constants of aluminum. Phys Rev 175(3): 955-962. https://doi.org/10.1103/ PhysRev.175.955

[44] Kube CM (2016) Elastic anisotropy of crystals. AIP Adv. h ttps://doi.org/10.1063/1.4962996

[45] Ledbetter HM (1984) Monocrystal polycrystal elastic-constants of a stainless-steel. Phys Status Solidi A 85(1):89-96. https://doi.org/10.1002/pssa.2210850111

[46] Kavyani M, Ebrahimi GR, Sanjari M, Haghshenas M (2016) Texture evaluation in warm deformation of an extruded $\mathrm{Mg}$ 6Al-3Zn alloy. J Magnes Alloy 4(2):89-98. https://doi.org/ 10.1016/j.jma.2016.05.002

[47] Jakubowska D, Zdunek J, Kulczyk M, Mizera J, Kurzydlowski KJ (2015) Microstructure and texture of hydrostatic extrusion deformed Ni single crystals and polycrystal. Adv Mater Sci Eng. https://doi.org/10.1155/2015/613839

[48] Bazarnik P, Romelczyk B, Kulczyk M, Lewandowska M (2013) The strength and ductility of 5483 aluminium alloy processed by various SPD methods. Light Metals Technol. h ttps://doi.org/10.4028/www.scientific.net/MSF.765.423

[49] Bohlen J, Yi SB, Swiostek J, Letzig D, Brokmeier HG, Kainer KU (2005) Microstructure and texture development during hydrostatic extrusion of magnesium alloy AZ31. Scr 
Mater 53(2):259-264. https://doi.org/10.1016/j.scriptamat.2 005.03 .036

[50] Maj P, Adamczyk-Cieslak B, Mizera J, Pachla W, Kurzydlowski KJ (2014) Microstructure and mechanical properties of duplex stainless steel subjected to hydrostatic extrusion. Mater Charact 93:110-118. https://doi.org/10.1016/j.matcha r.2014.03.017

[51] Clayton JD (2011) Nonlinear mechanics of crystals, 1st edn. Netherlands, Springer

[52] Kalisch J, Gluge R (2015) Analytical homogenization of linear elasticity based on the interface orientation distribution: a complement to the self-consistent approach. Compos Struct 126:398-416. https://doi.org/10.1016/j.compstruct.20 15.02 .072

[53] Kohlhauser C, Hellmich C (2012) Determination of Poisson's ratios in isotropic, transversely isotropic, and orthotropic materials by means of combined ultrasonicmechanical testing of normal stiffnesses: application to metals and wood. Eur J Mech a-Solid 33:82-98. https://doi. org/10.1016/j.euromechsol.2011.11.009

[54] Kuo CG, Hsu CY, Chen JH, Lee PW (2017) Discharge current effect on machining characteristics and mechanical properties of aluminum alloy 6061 workpiece produced by electric discharging machining process. Adv Mech Eng. h ttps://doi.org/10.1177/1687814017730756

[55] Arooj S, Shah M, Sadiq S, Jaffery SHI, Khushnood S (2014) Effect of current in the EDM machining of aluminum 6061 T6 and its effect on the surface morphology. Arab J Sci Eng 39(5):4187-4199. https://doi.org/10.1007/s13369-014-1020$\mathrm{Z}$

[56] Demellayer R, Richard J (2013) High precision electro discharge machining of molybdenum and tungsten. Proc Cirp 6:89-94. https://doi.org/10.1016/j.procir.2013.03.030

[57] ASM Hanbook Committee (1990) Properties of wrought aluminum and aluminum alloys. In: Properties and selection: nonferrous alloys and special-purpose materials, vol 2. ASM International, pp 683-687. https://doi.org/10.31399/asm.hb. v02.a0001060

[58] Balakirev FF, Ennaceur SM, Migliori RJ, Maiorov B, Migliori A (2019) Resonant ultrasound spectroscopy: The essential toolbox. Rev Sci Instrum 90(12):121401. https://d oi.org/10.1063/1.5123165

[59] Luo YK, Lin SZ, Fobes DM, Liu ZQ, Bauer ED, Betts JB, Migliori A, Thompson JD, Janoschek M, Maiorov B (2018) Anisotropic magnetocrystalline coupling of the skyrmion lattice in MnSi. Phys Rev B 97(10):104423. https://doi.org/ 10.1103/PhysRevB.97.104423

[60] Luo Y, Lin S-Z, Leroux M, Wakeham N, Fobes DM, Bauer ED, Betts JB, Thompson JD, Migliori A, Janoschek M, Maiorov B (2020) Skyrmion lattice creep at ultra-low current densities. Commun Mater 1(1):83. https://doi.org/10.1038/ s43246-020-00083-1

[61] Maiorov B, Betts JB, Soderlind P, Landa A, Hernandez SC, Saleh TA, Freibert FJ, Migliori A (2017) Elastic moduli of delta-Pu-239 reveal aging in real time. J Appl Phys 121(12):9. https://doi.org/10.1063/1.4978509

[62] Pantea C, Rickel DG, Migliori A, Leisure RG, Zhang JZ, Zhao YS, El-Khatib S, Li BS (2005) Digital ultrasonic pulseecho overlap system and algorithm for unambiguous determination of pulse transit time. Rev Sci Instr. https://doi.org/ $10.1063 / 1.2130715$

[63] Sturtevant BT, Pantea C, Sinha DN (2013) Evaluation of the transmission line model for couplant layer corrections in pulse-echo measurements. IEEE Trans Ultrason Ferroelectr Freq Control 60(5):943-953. https://doi.org/10.1109/tuffc.2 013.2651

[64] Press WH, Teukolsky SA, Vetterling WT, Flannery BP (1992) Numerical recipes in C, 2nd edn. Cambridge University Press, Cambridge

[65] Wenk HR, Lutterotti L, Vogel S (2003) Texture analysis with the new HIPPO TOF diffractometer. Nucl Instrum Meth A 515(3):575-588. https://doi.org/10.1016/j.nima.2003.05.001

[66] Vogel SC, Hartig C, Lutterotti L, Von Dreele RB, Wenk HR, Williams DJ (2004) Texture measurements using the new neutron diffractometer HIPPO and their analysis using the Rietveld method. Powder Diffr 19(1):65-68. https://doi.org/ 10.1154/1.1649961

[67] Lisowski PW, Schoenberg KF (2006) The Los Alamos neutron science center. Nucl Instrum Meth A 562(2):910-914. https://doi.org/10.1016/j.nima.2006.02.178

[68] Takajo S, Vogel SC (2018) Determination of pole figure coverage for texture measurements with neutron time-offlight diffractometers. J Appl Crystallogr 51:895-900. http s://doi.org/10.1107/S1600576718007732

[69] Losko AS, Vogel SC, Reiche HM, Nakotte H (2014) A sixaxis robotic sample changer for high-throughput neutron powder diffraction and texture measurements. J Appl Crystallogr 47:2109-2112. https://doi.org/10.1107/ S1600576714021797

[70] Lutterotti L, Matthies S, Wenk HR, Schultz AS, Richardson JW (1997) Combined texture and structure analysis of deformed limestone from time-of-flight neutron diffraction spectra. J Appl Phys 81(2):594-600. https://doi.org/10.1063/ 1.364220

[71] Rietveld HM (1969) A profile refinement method for nuclear and magnetic structures. J Appl Crystallogr 2:65-000. http s://doi.org/10.1107/S0021889869006558

[72] Wenk HR, Lutterotti L, Vogel SC (2010) Rietveld texture analysis from TOF neutron diffraction data. Powder Diffr 25(3):283-296. https://doi.org/10.1154/1.3479004 
[73] Hill R (1965) Continuum micro-mechanics of elastoplastic polycrystals. J Mech Phys Solids 13(2):89-000. https://doi. org/10.1016/0022-5096(65)90023-2

[74] Tomé CN, Lebensohn RA (2020) Polycrystal thermo-elasticity revisited: theory and applications. Comptes Rendus Mécanique. https://doi.org/10.5802/crmeca.18

[75] Woo CH (1985) Polycrystalline effects on irradiation creep and growth in textured zirconium. $\mathrm{J}$ Nucl Mater 131(2-3):105-117. https://doi.org/10.1016/0022-3115(85)9 0449-0

[76] Mura T (1987) Micromechanics of defects in solids. Springer, Dordrecht

[77] Eshelby JD (1957) The determination of the elastic field of an ellipsoidal inclusion, and related problems proceedings of the royal society of London Series a-mathematical and physical sciences 241(1226):376-396

[78] Simmons G, Wang H (1971) Single crystal elastic constants and calculated aggregate properties. MIT Press, Cambridge, Massachusetts

[79] Raju S, Sivasubramanian K, Mohandas E (2002) The high temperature bulk modulus of aluminium: an assessment using experimental enthalpy and thermal expansion data.
Solid State Commun 122(12):671-676. https://doi.org/10.1 016/S0038-1098(01)00517-8

[80] Thomsen L (1986) Weak elastic-anisotropy. Geophysics 51(10):1954-1966. https://doi.org/10.1190/1.1442051

[81] Paterson DAP, Ijomah W, Windmill JFC (2018) Elastic constant determination of unidirectional composite via ultrasonic bulk wave through transmission measurements: a review. Prog Mater Sci 97:1-37. https://doi.org/10.1016/j. pmatsci.2018.04.001

[82] Neighbours JR (1954) An approximation method for the determination of the elastic constants of single crystals. J Acoust Soc Am 26(5):865-869. https://doi.org/10.1121/1. 1907431

[83] Neighbours JR, Smith CS (1950) An approximation method for the determination of the elastic constants of cubic single crystals. J Appl Phys 21(12):1338-1339. https://doi.org/10. 1063/1.1699601

Publisher's Note Springer Nature remains neutral with regard to jurisdictional claims in published maps and institutional affiliations. 